GLOBAL EUROPE - BASEL PAPERS ON EUROPE IN A GLOBAL PERSPECTIVE

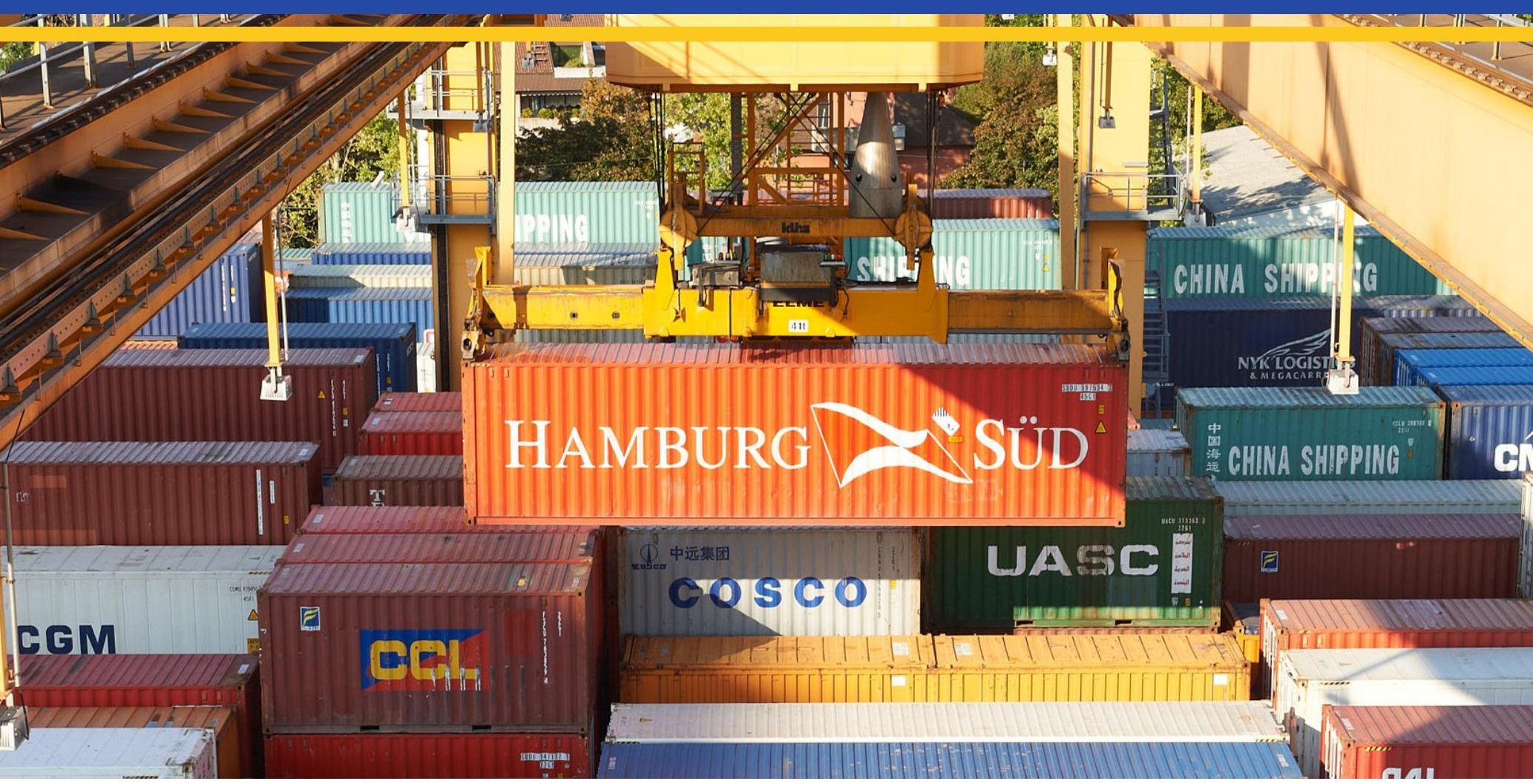

NR. 104

\title{
TANJA KLEIN
}

Die Gemeinsame Sicherheits- und Verteidigungspolitik in der Krise? Nationale Rollenkonzepte und Souveränität als Herausforderung europäischer Handlungsfähigkeit 
ISBN-13: 978-3-905751-26-0

ISBN-10: 3-90575 1-26-7

\section{(c) 2014}

\section{HERAUSGEBER}

Prof. Dr. Madeleiner Herren-Oesch, Direktorin

Dr. Isabella Löhr, Redaktion

Europainstitut der Universität Basel

Institute for European Global Studies

Gellertstrasse 27, Postfach

$\mathrm{CH}-4020$ Basel

\section{EUROPAINSTITUT BASEL}

Das Europainstitut Basel (EIB) ist ein dem Rektorat unterstelltes Forschungszentrum der Universität Basel. Als interdisziplinäres Institute for European Global Studies bündelt es Forschung und Lehre in einem Bereich, den die Universität Basel als einen der strategischen Schwerpunkte für die kommenden Leistungsperioden festgelegt hat. Das Europainstitut führt die etablierte Tradition der Auseinandersetzung mit europäischen Integrationsprozessen in Lehre und Forschung weiter und bietet auf diese Thematik spezialisierte Studiengänge sowie Weiterbildungskurse und Serviceleistungen an. 


\section{TANJA KLEIN}

Die Gemeinsame Sicherheits- und Verteidigungspolitik in der Krise? Nationale Rollenkonzepte und Souveränität als Herausforderung europäischer Handlungsfähigkeit

\section{KEYWORDS}

Common Security and Defence Policy - foreign policy role conceptions - sovereignty - actorness - identity

\section{ABSTRACT}

The objective of this paper is to identify sources of the challenges faced by the Common Security and Defence Policy (CSDP) of the European Union. Based on an actorness approach, the paper offers a systematic and theory-driven framework for the analysis of these challenges. Their investigation is based on publications of thirteen think tanks that were published in the context of the December summit of the European Council in 2013. The paper identifies two main sources at a national level. First, it shows that the significance of national sovereignty in the sensitive domain of security and defence policy is an obstacle to the development of the CSDP. Second, the analysis reveals that foreign policy role conceptions are a fundamental factor: different role conceptions of member states result in a lack of mutual understanding, in missing trust, and finally, in the unwillingness to transfer actorness to the EU level despite a common set of basic interests and values. Yet, the political will of member states will be decisive for the future development of the CSDP.

\section{TANJA KLEIN, M.A.}

Tanja Klein is research and teaching assistant at the Institute for European Global Studies at the University of Basel and PhD candidate in political science. She studied public administration/European studies at the Universities of Münster and Twente and public policy at the University of Potsdam. Her research focuses on foreign policy analysis and right-wing populist movements in Western Europe. In her PhD thesis, she is working on the positions of member states in the Common Security and Defence Policy of the European Union. 


\section{Inhalt}

1. Einleitung

2. Theoretischer und methodischer Hintergrund

2.1 Forschungsstand und Quellen

2.2 Theoretischer Ansatz

2.2.1 Actorness

2.2.2 Aussenpolitische Rollenkonzepte

2.2.3 Souveränität

2.3 Zwischenfazit

3. Die Gemeinsame Sicherheits- und Verteidigungspolitik (GSVP)

3.1 Entwicklung

3.2 Akteure und Entscheidungsprozesse

3.3 Missionen und Operationen

3.4 Zwischenfazit

\section{Herausforderungen}

4.1 Sicherheits- und verteidigungspolitische actorness der EU

4.2 Souveränitätskonzepte der Mitgliedstaaten

4.3 Rollenkonzepte der Mitgliedstaaten

4.4 Zwischenfazit

5.1 Der Dezembergipfel 2013

5.2 Reformvorschläge 


\section{Abkürzungsverzeichnis}

\begin{tabular}{|l|l|}
\hline Coreper & Ausschuss der Ständigen Vertreter (Comité des représentants permanents) \\
\hline EDA & European Defence Agency \\
\hline EEAS & European External Action Service \\
\hline ESS & Europäische Sicherheitsstrategie \\
\hline ESVP & Europäische Sicherheits- und Verteidigungspolitik \\
\hline EU & Europäische Union \\
\hline EUMC & European Union Military Committee \\
\hline EUMS & Military Staff of the European Union \\
\hline GASP & Gemeinsame Aussen- und Sicherheitspolitik \\
\hline GSVP & Gemeinsame Sicherheits- und Verteidigungspolitik \\
\hline NATO & North Atlantic Treaty Organization \\
\hline P\&S & Pooling \& Sharing \\
\hline PSK/COPS & Politisches und Sicherheitspolitisches Komitee (Comité politique et de sécurité) \\
\hline SSZ & Ständige Strukturierte Zusammenarbeit \\
\hline UNO & Vereinte Nationen \\
\hline
\end{tabular}




\section{Einleitung}

„Jenseits der Turbulenzen um die Währung wird die Europäische Union von einer zweiten Krise geplagt, die Bedeutung und Einfluss der Gemeinschaft in der Welt weiter empfindlich schmälern könnte: Die Gemeinsame Sicherheits- und Verteidigungspolitik (GSVP) der EU steht vor dem Scheitern. Mit Einsätzen wie gegen Piraten vor dem Horn von Afrika hat sich die EU zwar ein wenig Ansehen erworben. Doch sie ist weit entfernt von ihrem eigentlichen Ziel, einen wichtigen Baustein in einer globalen Sicherheitsarchitektur abzugeben."

Fünfzehn Jahre nach ihrer Gründung kann die Gemeinsame Sicherheits- und Verteidigungspolitik (GSVP) der Europäischen Union bereits auf über dreissig abgeschlossene und laufende Missionen und Operationen zurückblicken. ${ }^{2}$ Die Spannbreite reicht von der Rechtsstaatsmission EULEX im Kosovo über die Polizeimission EUPOL DR Kongo bis hin zur Marinemission Atalanta (EU-NAVOR Somalia), die am Horn von Afrika den Schutz humanitärer Hilfslieferungen und der Seefahrt gewährleisten und die Piraterie bekämpfen soll. Ist diese beeindruckende Zahl aber nur die halbe Wahrheit? Vor dem Dezembergipfel der Staats- und Regierungschefs der EU 2013 diagnostizierten Kommentatoren einen Stillstand, das Scheitern oder gar den, Tod' der GSVP. Tatsächlich kommt der GSVP seit einigen Jahren nur wenig politische Aufmerksamkeit zu: „Die zögerliche Herangehensweise von EU-Mitgliedern ist dabei auch im Kontext der europapolitischen Gesamtlage zu sehen: Sicherheitspolitik wird derzeit der Rettung des Euro und der Wirtschaftspolitik untergeordnet." ${ }^{3}$

Vor diesem Hintergrund weckte der Dezembergipfel 2013 des Europäischen Rates Hoffnungen zumindest auf ein Aufbruchsignal: Erstmals seit fünf Jahren wollten sich die Staats- und Regierungschefs wieder persönlich mit der GSVP auseinandersetzen. Vorbereitet wurde der Gipfel unter anderem durch Berichte der Europäischen Kommission und der Hohen Vertreterin der EU für Aussen- und Sicherheitspolitik sowie durch ein Treffen der Verteidigungsminister im September 2013.4 Dies löste eine grössere Zahl von Veröffentlichungen über die GSVP durch Think Tank sowie Medienberichte aus. Eine Analyse dieser

\footnotetext{
1 Winter, Martin: Mit Sicherheit nichts zu machen. Im Schatten der Währungskrise stevert die EU auf den Kollaps ihrer gemeinsamen Verteidigungspolitik zu, in: Süddeutsche Zeitung, 7.5.2013, 7.

2 Die Europäische Sicherheits- und Verteidigungspolitik (ESVP) wurde im Vertrag von Lissabon in Gemeinsame Sicherheits- und Verteidigungspolitik (GSVP) umbenannt. Für das bessere Leseverständnis wird einheitlich die Abkürzung GSVP verwendet.

3 Keller, Patrick/Möller, Almut/Sinjen, Svenja/Varwick, Johannes: Zivilmacht mit Zähnen. Deutsche Vorschläge für eine Neubelebung der GSVP, in: Internationale Politik, März/April 2012, 82. Vgl. auch Arbeitskreis Internationale Sicherheitspolitik: Ein Kompass für die GSVP, Internationale Politikanalyse, Friedrich-Ebert-Stiftung, April 2012, 3.
}

4 Ashton, Catherine: Preparing the December 2013 European Council on Security and Defence, Final Report by the High Representative/Head of the EDA on the Common Security and Defence Policy, Brüssel, 15.10.2013; European Commission: A New Deal for European Defence. Towards a More Competitive and Efficient Defence and Security Sector. Communication from the Commission to the European Parliament, the Council, the European Economic and Social Committee and the Committee of the Regions, COM (2013) 542. 
Publikationen ermöglicht einen aktuellen Einblick in die Herausforderungen, vor denen die GSVP steht.

Das vorliegende Paper geht gleichzeitig einen Schritt weiter, indem es ein theoretisch verankertes Analyseschema entwickelt, das eine Untersuchung der Hauptdiagnose der Think Tank-Publikationen ermöglicht: Die GSVP stecke in der Krise. Ausgehend von dieser Diagnose stellt das Paper die Ursachen der aktuellen Probleme in den Blickpunkt: Wie lässt sich diese Krise verstehen? Wo liegen die Wurzeln der Herausforderungen, vor denen die GSVP heute steht? Die GSVP kann nur dann erfolgreich sein, wenn die EU über eine ausreichende sicherheits- und verteidigungspolitische Handlungsfähigkeit verfügt. Das Paper analysiert diese Handlungsfähigkeit aufbauend auf dem actorness-Ansatz von Jupille/Caporaso 5 systematisch anhand der sicherheits- und verteidigungspolitischen actorness der EU. Es wird die These aufgestellt, dass es der EU an sicherheits- und verteidigungspolitischer actorness mangelt. Angenommen wird, dass diese fehlende actorness auf den aus aussenpolitischen Rollenkonzepten und Souveränitätskonzepten resultierenden Unwillen der Mitgliedstaaten zurückzuführen ist, Handlungsfähigkeit im sensiblen Bereich der Sicherheit und Verteidigung an die EU abzugeben. Dies verhindert einen echten Neustart der GSVP und ermöglicht nur kleine Fortschritte. Eine solche Rückführung der sicherheits- und verteidigungspolitischen actorness der EU auf nationale Rollenkonzepte und Souveränitätsvorstellungen trägt dem intergouvernementalen Charakter der GSVP Rechnung und erlaubt eine Betrachtung, die die Analyseebenen Staat und EU miteinander verbindet.

Der erste Teil des Papers behandelt den theoretischen und methodischen Hintergrund der Arbeit und führt das Analyseschema ein. Im Anschluss folgt eine kurze Darstellung der Grundzüge der GSVP, bevor anhand des Analyserasters aktuelle Herausforderungen an die GSVP näher untersucht werden. Hierauf aufbavend stellt das folgende Kapitel Vorschläge für die zukünftige Gestaltung der GSVP in den Blickpunkt. In den abschliessenden Schlussfolgerungen werden die Ergebnisse zusammengeführt und es wird diskutiert, ob sich die GSVP nach dem Dezembergipfel 2013 in einer Krise befindet oder vielmehr einen Neustart vor Augen hat.

\section{Theoretischer und methodischer Hintergrund}

Im Folgenden werden zunächst der Forschungsstand und mögliche Anknüpfungspunkte dargestellt. Daran schliessen sich eine Vorstellung der verwendeten Quellen sowie des Analyserahmens an.

5 Vgl. Jupille, Joseph/Caporaso, James A.: States, Agency, and Rules: The European Union in Global Environmental Politics, in: Rhodes, Carolyn (Hg.): The European Union in the World Community, Boulder/London 1998, 21 3-230. 


\subsection{Forschungsstand und Quellen}

Die GSVP ist ein aus politikwissenschaftlicher Perspektive intensiv erforschtes Politikfeld. So finden sich zahlreiche grundlegende Darstellungen zur Gemeinsamen Aussen- und Sicherheitspolitik (GASP) und zu den Aussenbeziehungen der EU im Allgemeinen, die Betrachtungen der GSVP beinhalten. ${ }^{\circ}$ Daneben existieren Überblicksdarstellungen zur GSVP und zur institutionellen Entwicklung der GASP/GSVP.7 Einzelne Aspekte, die spezielle Aufmerksamkeit erhalten, sind unter anderem die Europäische Sicherheitsstrategie (ESS), das Verhältnis zwischen EU und NATO sowie die GSVP-Operationen und deren Erfolg bzw. Misserfolg. Zudem existieren erste Bilanzen zur GSVP und ihren Missionen. ${ }^{8}$

Wenig erforscht sind demgegenüber die GSVP-Politiken der Mitgliedstaaten. Vor allem vergleichende Betrachtungen nationaler GSVP-Politiken bleiben Mangelware oder sind wenig systematisch. ${ }^{9}$ Werden mitgliedstaatliche Politiken untersucht, so stehen in der Regel die ,Grossen Drei', Deutschland, Frankreich und Grossbritannien im Fokus. Darüber hinaus fällt auf, dass Veröffentlichungen über die GSVP häufig nicht oder nur oberflächlich theoretisch verankert sind. Dies ist einerseits darauf zurückzuführen, dass viele aktuelle Veröffentlichungen aus wissenschaftlichen Think Tanks stammen. Solche policy papers, zum Beispiel des European Union Institute for Security Studies, der Stiftung Wissenschaft und Politik oder des Centre for European Reform liefern jedoch zeitnahe Darstellungen aktueller Missionen, Entwicklungen und Herausforderungen und bilden eine Basis für die folgende Analyse. Andererseits ist die GSVP ein Forschungsgegenstand, der mit den klassischen politikwissenschaftlichen Theorien der europäischen Integration und der Internationalen Beziehungen nicht immer leicht zu greifen ist. Da die GSVP bis heute

6 Einen Überblick zur GASP einschliesslich GSVP bietet bspw. Algieri, Franco: Die Gemeinsame Außen- und Sicherheitspolitik der EU, Wien 2010.

7 So beispielsweise Diedrichs, Udo: Die Gemeinsame Sicherheits- und Verteidigungspolitik der EU, Wien 2012.

8 Eine Bilanz der ersten zehn Jahre GSVP findet sich bei Grevi, Giovanni/Helly, Damien/Keohane, Daniel (Hg.): European Security and Defence Policy. The First Ten Years (1999-2000), The European Union Institute for Security Studies, Paris 2009. Die Missionen und Operationen analysieren Asseburg, Muriel/Kempin, Ronja (Hg.): Die EU als strategischer Akteur in der Sicherheits- und Verteidigungspolitik? Eine systematische Bestandsaufnahme von ESVP-Missionen und -Operationen, Stiffung Wissenschaft und Politik, S32, Dezember 2009.

9 Darstellungen von GASP/GSVP-Politiken finden sich bei Müller-Brandeck-Bocquet, Gisela (Hg.): Europäische Außenpolitik. GASP- und ESVP-Konzeptionen ausgewählter EU-Mitgliedstaaten, Baden-Baden 2002; Ehrhart, Hans-Georg (Hg.): Die Europäische Sicherheits- und Verteidigungspolitik. Positionen, Perzeptionen, Probleme, Perspektiven, Baden-Baden 2002. Darüber hinaus existieren Sammelbände mit Texten zu nationalen Aussenpolitiken einschliesslich der GASP, z.B. Hill, Christopher: The Actors in Europe's Foreign Policy, London 1996. Schliesslich bestehen Veröffentlichungen zu den GSVP- und GASP-Politiken einzelner Staaten. So behandelt Tonra die Europäisierung nationaler Aussenpolitiken anhand der Fallstudien Dänemark, Irland und Niederlande. Vgl. Tonra, Ben: The Europeanisation of National Foreign Policy. Dutch, Danish and Irish Foreign Policy in the European Union, Aldershot 2001 . Larsen erstellt eine Einzelfallstudie für Dänemark in der GASP. Vgl. Larsen, Henrik: Danish CFSP Policy in the Post-Cold War Period: Continuity or Change?, in: Cooperation and Conflict, 35 (2000) 1, 37-63. Eine Analyse der deutschen EPZ- und GASP-Politiken findet sich bei Schmalz, Uwe: Deutschlands europäisierte Außenpolitik. Kontinuität und Wandel deutscher Konzepte zur EPZ und GASP, Wiesbaden 2003. Die GSVP-Politik Polens untersucht Frank, Cornelia: Zivilmacht trifft, instinktiven' Atlantiker: Deutschlands und Polens Interessen in der ESVP, in: Jäger, Thomas/Dylla, Daria W. (Hg.): Deutschland und Polen. Die europäische und internationale Politik, Wiesbaden 2008, 101-122. Eine weitere Analyse polnischer GSVPPolitik findet sich in Chappell, Laura: Poland in Transition: Implications for a European Security and Defence Policy, in: Contemporary Security Policy 31 (2010) 2, 225-248. 
massgeblich durch die Mitgliedstaaten bestimmt wird, bieten sich insbesondere (neo)realistische und intergouvernementale Ansätze an. ${ }^{10}$ Vor allem der Liberale Intergouvernementalismus nach Andrew Moravcsik wird häufig zur Analyse herangezogen. Institutionalistische Ansätze verweisen demgegenüber auf die zunehmende Überwindung klassischer intergouvernementaler Zusammenarbeit in der GASP, eine Zunahme der Handlungsfähigkeit der EU oder die Einbeziehung anderer Akteure, welche die GSVP jenseits ihrer intergouvernementalen Rolle mit anderen Politikfeldern und Akteuren verbindet. Zudem wird zunehmend auf konstruktivistische Fragen nach einer gemeinsamen strategischen Kultur und Sicherheitsidentität eingegangen. ${ }^{11}$

Die vorliegende Arbeit nutzł daher einerseits die zeitnahen Analysen aktueller Herausforderungen für die GSVP in Publikationen wissenschaftlicher Think Tanks. Sie bezieht Veröffentlichungen von insgesamt dreizehn Think Tanks ein, die im Umfeld des Europäischen Rats im Dezember 2013 erschienen sind. Dies ermöglicht einen umfassenden Einblick in aktuelle Herausforderungen an die GSVP, aber auch in mögliche Reformen. Ergänzł werden diese Publikationen durch entsprechende Medienberichte.

\begin{tabular}{|l|l|}
\hline $\begin{array}{l}\text { Centre for European Reform } \\
\text { EGMOntre for European Studies }\end{array}$ & $\begin{array}{l}\text { Institut de Relations Internationales et Stratégiques } \\
\text { Istituto Affari Internazionali } \\
\text { European Policy Centre }\end{array}$ \\
$\begin{array}{l}\text { European Union Institute for Security Studies } \\
\text { Friedrich-Ebert-Stiftung }\end{array}$ & $\begin{array}{l}\text { Real Institute Elcano } \\
\text { Stiftung Wissenschaft und Politik }\end{array}$ \\
Heritage Foundation & The Finnish Institute of International Affairs \\
\hline
\end{tabular}

Abb. 1: Think Tanks, deren Publikationen in die Analyse einbezogen wurden. ${ }^{12}$

Andererseits erfolgt anhand des actorness-Ansatzes eine Systematisierung der Herausforderungen. Um hierauf aufbauend eine nähere Analyse der Bedeutung der Mitgliedstaaten für die Handlungsfähigkeit zu ermöglichen, wird der actorness-Ansatz mit nationalen Souveränitäts- und Rollenkonzepten verbunden.

10 Eine neorealistische Betrachtung präsentiert Rynning, Sten: Realism and the Common Security and Defence Policy, in: Journal of Common Market Studies 49 (201 1) 1, 23-42.

11 Ein Beispiel für einen konstruktivistischen Ansatz bietet Giegerich, Bastian: European Security and Strategic Culture. National Responses to the EU's Security and Defence Policy, Baden-Baden 2006. Eine genavere Darstellung der Analyse der GSVP aus unterschiedlichen konzeptionellen und theoretischen Perspektiven findet sich bei Diedrichs (2012), 20-23.

12 Von der Friedrich-Ebert-Stiftung wurde ein Paper des Arbeitskreises, Internationale Sicherheitspolitik' aus dem Jahr 2012 genutzt, da dieses die jüngste Publikation zum Thema ist. 
Die Arbeit leistet so eine systematische und theoretisch verankerte Analyse aktueller Probleme und Herausforderungen der GSVP und bestätigt die Bedeutung der weiteren Untersuchung nationaler GSVP-Politiken.

\subsection{Theoretischer Ansatz}

Zunächst wird in den actorness-Ansatz eingeführt. Im Anschluss steht die Einwirkung aussenpolitischer Rollenkonzepte der Mitgliedstaaten und ihrer Souveränitätskonzepte auf die sicherheits- und verteidigungspolitische actorness der EU im Blickpunkt.

\subsubsection{Actorness}

Jupille/Caporaso entwickelten den hier verwendeten actorness-Ansatz ursprünglich für die Analyse europäischer Umweltpolitik. ${ }^{13}$ Er erweist sich aber auch für andere aussenpolitische Felder als gewinnbringend. Die Autoren gehen davon aus, dass drei grundlegende Denkrichtungen zu der Frage existieren, wie sich die Rolle der EU in den Aussenbeziehungen bestimmen lässt:

1) die EU als Sammlung von Staaten; ${ }^{14}$

2) die EU als polity oder sich entwickelnde polity;

3) die EU als entstehende Einheit, die unterschiedliche Themenfelder und Netzwerke umfasst und abhängig von Thema und Zeit einen wechselnden Grad an actorness aufweist. ${ }^{15}$

Anknüpfend an den dritten Aspekt entwickeln Jupille/Caporaso den actorness-Ansatz, der eine systematische Erfassung der Handlungsfähigkeit von Akteuren in der internationalen Politik ermöglicht. Sie gehen dabei von der konstruktivistischen Annahme aus, dass die Handlungsfähigkeit durch Interaktion konstituiert wird. Bezogen auf die EU wird somit gefragt, inwiefern die Union ein internationaler Akteur ist.

13 Die Ausführungen zum actorness-Konzept basieren auf Jupille/Caporaso (1998), 21 3-230. Ergänzend verwendet wurde eine Anwendung von Harnisch/Stahl auf die Aussenpolitik, vgl. Harnisch, Sebastian/Stahl, Bernhard: Einleitung: EU-Außenpolitik und Actorness, in: Stahl, Bernhard/Harnisch, Sebastian (Hg.): Vergleichende Außenpolitikforschung und nationale Identitäten. Die Europäische Union im Kosovo-Konflikt 1996-2008, Baden-Baden 2009, 15-29. Die Autoren analysieren mit Hilfe des actorness-Ansatzes die Kosovo-Politik der EU. Basierend auf einem vergleichenden Konzept, das die Positionen Deutschlands, Frankreichs, Griechenlands, Grossbritanniens und der Niederlande untersucht, wird über die actorness nach der kollektiven Handlungsfähigkeit der EU gefragt. Harnisch/Stahl sehen eine zentrale Rolle gesellschaftlicher Diskurse für das aussenpolitische Engagement der EU und versuchen, durch nationale Diskursformationen in Fallstudien identitäre Schnittmengen der nationalen Identitäten zu erkennen: „Nationalstaatliche Identitäten konditionieren die internationale Akteursschaft und damit die außenpolitische Handlungsfähigkeit der Europäischen Union.",

Harnisch, Sebastian/Stahl, Bernhard: Fazit: Die identitären Möglichkeitsräume der EU-Außenpolitik, in: Stahl, Bernhard/Harnisch, Sebastian (Hg.): Vergleichende Außenpolitikforschung und nationale Identitäten. Die Europäische Union im Kosovo-Konflikt 1996-2008, BadenBaden 2009, 278. Das Untersuchungskonzept wird dargestellt in Harnisch/Stahl (2009), 15-29. Die zentralen Ergebnisse der Fallstudien finden sich in Harnisch/Stahl (2009), 275-292.

14 Die EU ist hier nur dann ein ,kollektiver Akteur', wenn eine Interessenkonvergenz ihrer Einheiten, d.h. der Staaten besteht.

15 Vgl. Jupille/Caporaso (1998), 213-214. 
Welche Bedeutung hat der actorness-Ansatz aber für die GSVP? Hier ist anzunehmen, dass die GSVP nur dann erfolgreich agieren kann, wenn die EU eine ausreichende sicherheits- und verteidigungspolitische Handlungsfähigkeit aufweist. Somit bietet sich der actorness-Ansatz als Instrument zur systematischen Analyse der Herausforderungen an, vor denen die GSVP steht. Konkret werden in Kapitel vier die Herausforderungen an die GSVP den vier Komponenten Anerkennung, Kohärenz, Autorität und Autonomie zugeordnet, deren Existenz und Qualität Jupille/Caporaso als Grundlage der Handlungsfähigkeit beschreiben. Der Ansatz ermöglicht folglich eine detaillierte Erfassung der sicherheits- und verteidigungspolitischen actorness und somit der Handlungsfähigkeit der EU in der GSVP. Im Folgenden werden die vier Komponenten der actorness kurz eingeführt:

Kohärenz: Die Kohärenz als erste Komponente unterteilen Jupille/Caporaso in Ziel- und Wertekohärenz, prozedurale Kohärenz, taktische Kohärenz und outputKohärenz. "The final component is cohesion, or the degree to which an entity is able to formulate and articulate internally consistent policy preferences." 16 Aufbauend auf dieser Definition bezieht sich Zielkohärenz auf ähnliche oder komplementäre Ziele der Mitgliedstaaten, während taktische Kohärenz von leicht unterschiedlichen Zielen ausgeht, die jedoch durch Ausgleich, durch package deals und ähnliches überwunden werden können. Prozedurale Kohärenz bezeichnet gemeinsame Regeln und Abläufe. Einigen sich die Mitgliedstaaten auf eine gemeinsame Politik, wird schliesslich eine höhere outputKohärenz angenommen. In allen vier Kohärenz-Typen können Konflikte auftreten, die die Handlungsfähigkeit der EU negativ beeinflussen. Denkbar sind Konflikte sowohl auf Ebene der EU oder der Mitgliedstaaten (horizontal) als auch zwischen unterschiedlichen Ebenen (vertikal). ${ }^{17}$

Autonomie: Der Aspekt der Autonomie umfasst einerseits die institutionelle Unterscheidbarkeit und andererseits die Unabhängigkeit. Erstere bezieht sich auf die Existenz eigener Organisationseinheiten. Letztere demgegenüber definieren Jupille/Caporaso wie folgt: „By independence we mean that these institutions should make a difference, compared to the baseline expectation of a decentralized state system working on the basis of power and interest." ${ }^{18}$ Die EU muss nach dieser Sichtweise mehr sein als die Summe ihrer Teile. Sie formuliert nach eigenem Ermessen Ziele, legt die Entscheidungsfindung fest und implementiert Entscheidungen. ${ }^{19}$ Harnisch/Stahl ergänzen, dass neben Unterscheidbarkeit und Unabhängigkeit auch die „Verfügbarkeit außenpolitischer Ressourcen"20 ein wichtiger Teil der Autonomie sei.

\footnotetext{
16 Jupille/Caporaso (1998), 214.

17 Vgl. Jupille/Caporaso (1998), 21 8-220.

18 Jupille/Caporaso (1998), 217.

19 Vgl. Jupille/Caporaso (1998), 217-218.

20 Harnisch/Stahl (2009), 22.
} 
Autorität: Das dritte Element, die Autorität, umfasst die vertraglichen Grundlagen bzw. die rechtlichen Kompetenzen für das auswärtige Handeln der EU. Die Autorität leitet sich dabei von den Mitgliedstaaten ab: "Thus, to speak of the EU's authority is to think of authority delegated to EU institutions by nation states." 21

Anerkennung: Die vierte Komponente beinhaltet schliesslich die Anerkennung einer Einheit durch andere Akteure. Dabei kann es sich sowohl um eine de jure-Anerkennung (Anerkennung der Rechtspersönlichkeit durch Staaten/Organisationen, Mitgliedschaft in internationalen Organisationen) als auch um eine de facto-Anerkennung (Interaktion in der Praxis) handeln: "Recognition by others allows for presence in global politics, which, not surprisingly is the sine qua non of global actorhood."22

Bezieht man den aussenpolitischen Ansatz der actorness spezifisch auf die Sicherheits- und Verteidigungspolitik der EU, so ergibt sich zusammenfassend folgendes Bild:
Kohärenz,
Autonomie,
Autorität,
Anerkennung
Sicherheits- und verteidigungs-
politische actorness der EU /
Handlungsfähigkeit
Erfolg der GSVP

Abbildung 2: Die sicherheits- und verteidigungspolitische actorness der EU (eigene Darstellung).

Aus dem Ansatz Jupille/Caporasos lässt sich nicht ableiten, welche Faktoren die vier Elemente der sicherheits- und verteidigungspolitischen actorness beeinflussen. Sichtbar wird in der obigen Darstellung des aussenpolitischen actorness-Ansatzes aber die Bedeutung der Mitgliedstaaten. Da die GSVP das am stärksten intergouvernemental geprägte Politikfeld der EU ist, ist anzunehmen, dass Herausforderungen für die Handlungsfähigkeit der EU hier besonders stark auf mitgliedsstaatlicher Ebene zu finden sind. Die EU kann also nur dann über sicherheits- und verteidigungspolitische Kohärenz, Autorität und Autonomie verfügen, wenn die Mitgliedstaaten ihr diese zuerkennen. ${ }^{23}$ Daher werden zwei Fakłoren herangezogen, die auf nationaler Ebene auf die sicherheits- und verteidigungspolitische actorness der EU einwirken: aussenpolitische Rollenkonzepte und Souveränität.

\footnotetext{
21 Jupille/Caporaso (1998), 216.
}

22 Jupille/Caporaso (1998), 215. Vgl. zur Anerkennung Jupille/Caporaso (1998), 215-216; Harnisch/Stahl (2009), 20. Jupille/ Caporaso und Harnisch/Stahl weisen auf Probleme mit der de jure-Anerkennung hin, da die EU kein Staat ist. Harnisch/Stahl ergänzen, dass die Mitgliedstaaten nur in den vergemeinschafteten Bereichen (Europäische Gemeinschaft) eine Rechtspersönlichkeit der EU und hiermit deren Handlungskompetenz anerkannt hätten. Mit dem Vertrag von Lissabon wurde jedoch die Rechtspersönlichkeit für die EU eingeführt (Art. 47 EUV).

23 Harnisch/Stahl weisen darauf hin, dass eine Anerkennung durch Dritte auch von den Mitgliedstaaten abhängig ist. Eine Anerkennung durch Dritte könne erst dann erfolgen, wenn die Mitgliedstaaten der EU Autorität und Autonomie zuerkennen. Hier sehen Harnisch/ Stahl eine entscheidende Rolle für die nationalen Identitäten. Diese könnten für die EU-Institutionen die Möglichkeit zum Handeln schaffen. Dies wiederum führe zur Anerkennung der EU durch andere Akteure. Vgl. Harnisch/Stahl (2009), 23. 


\subsubsection{Aussenpolitische Rollenkonzepte}

In noch stärkerem Masse als andere Felder der europäischen Aussenbeziehungen wird die GSVP durch die Mitgliedstaaten bestimmt. Wie aber lassen sich nationale GSVP-Politiken verstehen? Einen vielversprechenden Ansatz bieten aussenpolitische Rollenkonzepte. Rollenkonzepte stammen ursprünglich aus der Soziologie und Psychologie, werden aber seit den 1970er Jahren auch in der Aussenpolitikanalyse verwendet. ${ }^{24}$ Aussenpolitische Rollenkonzepte sind relativ stabil. Geformt werden sie einerseits durch das Selbstbild eines Landes (ego) und andererseits durch die Erwartungen, die von aussen, zum Beispiel durch andere Staaten, an das Land gerichtet werden (alter). ${ }^{25}$ Ein Staat kann in den internationalen Beziehungen gleichzeitig verschiedene Rollen einnehmen, die sich auch widersprechen können (Rollenkonflikt). ${ }^{26}$ Das aussenpolitische Rollenkonzept eines Landes schafft einen Rahmen für die tatsächliche Aussenpolitik. Es setzł Grenzen für ein akzeptiertes aussenpolitisches Handeln, bietet aber auch eine gewisse Flexibilität. Aussenpolitische Rollenkonzepte bilden somit auch den Rahmen für die GSVP-Politiken der Mitgliedstaaten. Es ist anzunehmen, dass Rollenkonzepte einen Einfluss darauf haben, in welchem Masse ein Mitgliedstaat die sicherheits- und verteidigungspolitische Autorität und Autonomie der EU anerkennt. Nur ähnliche Rollenkonzepte ermöglichen eine Kohärenz der sicherheits- und verteidigungspolitischen Positionen der Mitgliedstaaten. In der Folge beeinflussen aussenpolitische Rollenkonzepte die sicherheits- und verteidigungspolitische actorness der EU und ihre Handlungsfähigkeit.

\subsubsection{Souveränität}

Souveränität bezeichnet nach innen die Selbstbestimmung eines Staates und nach aussen die Unabhängigkeit von anderen Staaten und ist somit "[...] the supreme legal authority of the national to give and enforce the law within a certain territory and, in consequence, independence from the authority of any other nation and equality with it under international law". ${ }^{27}$ Sicherheits- und Verteidigungspolitik bildet einen Kern nationaler Souveränität. Im Zuge der europäischen Integration übertragen die Mitgliedstaaten Teile ihrer Souveränität auf die EU. Die besondere Bedeutung der Sicherheits- und Verteidigungspolitik als Kern nationaler Souveränität stellt somit eine grosse Herausforderung für eine GSVP dar und hat Auswirkungen auf die sicherheits- und verteidigungspolitische actorness der EU. In welchem Masse die Mitgliedstaaten

24 Vgl. Biddle, Peter: Recent Developments in Role Theory, in: Annual Review of Sociology, 1986, 67-92.

25 Vgl. Holsti, Kal J.: National Role Conceptions in the Study of Foreign Policy, in: International Studies Quarterly, 14 (1970) 3, 241-247. Holsti konzentriert sich auf das ego. Demgegenüber betonen Jönsson/Westerlund auch die Bedeutung des alter. Vgl Jönsson, Christer/Westerlund, Ulf: Role Theory in Foreign Policy Analysis, in: Jönsson, Christer (Hg.): Cognitive Dynamics and International Politics, London 1982, 149

26 Vgl. Gaupp, Peter: Staaten als Rollenträger. Die Rollentheorie als Analyse-Instrument von Außenpolitik und internationalen Beziehungen, Liebefeld/Bern 1983, 78-86, 148-157.

27 Morgenthau, Hans: Politics among Nations. The Struggle for Power and Peace, New York 1967, 305. 
Souveränität auf die EU übertragen, wird auch durch die nationalen Rollenkonzepte beeinflusst.

\subsection{Zwischenfazit}

Zusammenfassend wird im Folgenden davon ausgegangen, dass die aussenpolitischen Rollenkonzepte der Mitgliedstaaten und ihr Souveränitätsverständnis die sicherheits- und verteidigungspolitische actorness der EU beeinflussen. Diese ist ausschlaggebend für die Handlungsfähigkeit der GSVP und somit für deren Erfolg oder Misserfolg. Das Schema ermöglicht eine systematische Analyse der Herausforderungen, vor denen die GSVP steht und zeigt theorienbasiert mögliche Ursachen auf nationaler Ebene für eine Krise der GSVP auf.

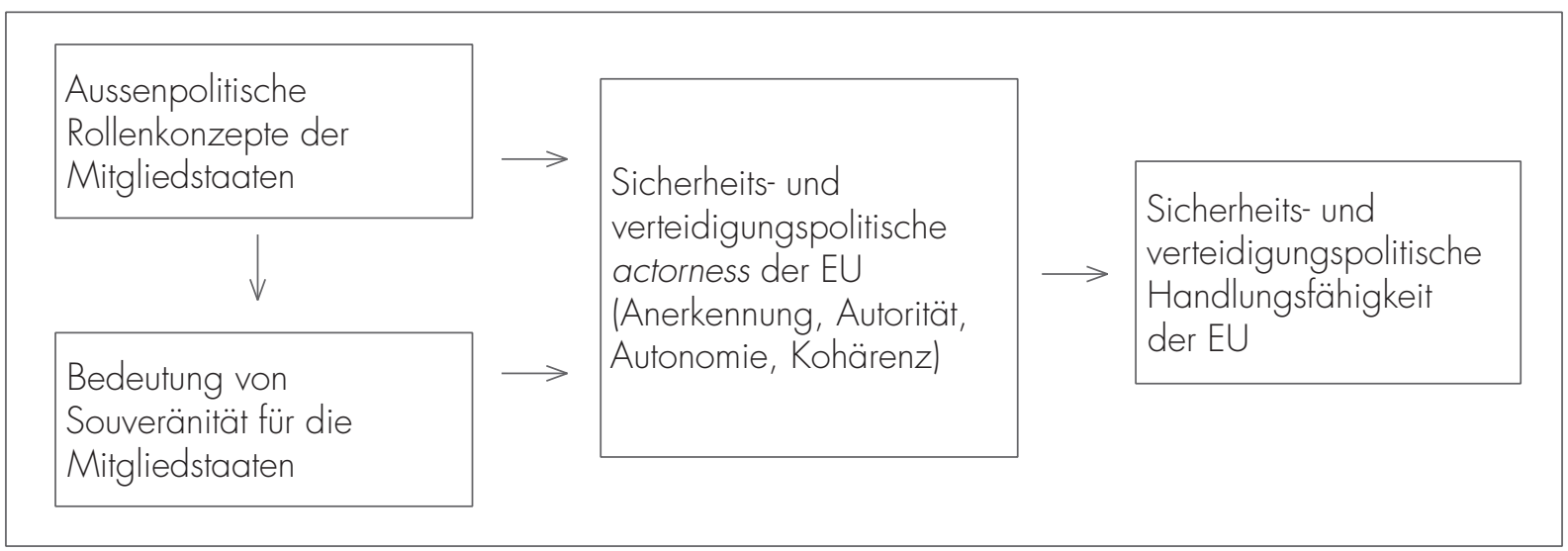

Abbildung 3: Die Einwirkung von Rollenkonzepten und Souveränität auf die sicherheits- und verteidigungspolitische actorness der EU (eigene Darstellung).

\section{Die Gemeinsame Sicherheits- und Verteidigungspolitik (GSVP)}

Die GSVP ist ein integraler Bestandteil der Gemeinsamen Aussen- und Sicherheitspolitik der EU, weist aber teils eigene Regeln, Verfahren und Organisationseinheiten auf. Daher werden im Folgenden die Entwicklung, die Akteure und Entscheidungsprozesse der GSVP überblicksweise dargestellt, bevor abschliessend kurz auf die Missionen und Operationen eingegangen wird.

\subsection{Entwicklung}

Ideen zu einer europäischen Zusammenarbeit in sicherheits- und verteidigungspolitischen Fragen entstanden bereits in den 1950er Jahren. Erste Versuche zur Gründung einer Europäischen Verteidigungsgemeinschaft scheiterten jedoch im Jahre 1954 an der Ablehnung durch die französische Nationalver- 
sammlung. ${ }^{28}$ In den 1970er Jahren begannen die Mitgliedstaaten schliesslich mit einer aussenpolitischen Kooperation im Rahmen der Europäischen Politischen Zusammenarbeit. Die Verteidigungspolitik blieb hiervon jedoch ausgeschlossen. ${ }^{29}$

Nach dem Ende des Kalten Krieges sahen sich die Europäer mit einer veränderten Umwelt konfrontiert, der die bisherigen Mechanismen der aussenpolitischen Kooperation nicht mehr gerecht wurden. So mussten die Mitgliedstaaten nicht nur erkennen, dass sie im Golfkrieg unkoordiniert reagierten und wenig Einfluss auf US-amerikanische Entscheidungen ausüben konnten, sondern auch, wie unvorbereitet der Kontinent auf den Ausbruch der Konflikte auf dem Balkan war. Als Reaktion wurde im Vertrag von Maastricht (1993) die Gemeinsame Aussen- und Sicherheitspolitik (GASP) eingeführt. ${ }^{30}$

Trotz der Gründung der GASP und ihrer späteren Stärkung ${ }^{31}$ waren die EU-Staaten aber selbst in ihrer unmittelbaren Nachbarschaft, im ehemaligen Jugoslawien, weiterhin auf die Hilfe der USA angewiesen. Gleichzeitig zeigten sich die Europäer zunehmend irritiert über das Agieren des amerikanischen Partners auf dem Balkan. "The Kosovo war demonstrated the disparity between European and American capabilities and approaches even more sharply." ${ }^{22}$ Die Erfahrungen mit der britisch-französischen Zusammenarbeit während der Konflikte waren demgegenüber positiv. Zudem sahen sich die Europäer mit der amerikanischen Forderung nach einer stärkeren Lastenteilung konfrontiert. ${ }^{33}$ Dieser Hintergrund eröffnete die Möglichkeit für den Gipfel von Saint Malo (1998). Während dieses Treffens des britischen Premierministers Tony Blair und des französischen Präsidenten Jacques Chirac kamen die beiden führenden europäischen Militärmächte überein, eine europäische Verteidigungskomponente zu unterstützen. ${ }^{34}$ In der Gipfelerklärung bekräftigen beide Staaten:

"The European Union needs to be in a position to play its full role on the international stage. [...] To this end, the Union must have the capacity for autonomous action, backed up by credible military forces, the means to decide to use them, and a readiness to do so, in order to respond

28 Vgl. zur Geschichte der GSVP Diedrichs (2012), 27-40.

29 Die Aussenminister der Mitgliedstaaten trafen sich ab 1970 vierteliährlich zu Gesprächen. Die EPZ war ein intergouvernementales Politikfeld und befand sich zunächst ausserhalb der Verträge. 1986 wurde sie durch die Einheitliche Europäische Akte in die Verträge integriert. Vgl. Wallace, William: Foreign and Security Policy. The Painful Path from Shadow to Substance, in: Wallace, Helen/Wallace, William/Pollack, Mark A. (Hg.): Policy-Making in the European Union, Oxford 2005, 433-435.

30 Vgl. Diedrichs (2012), 27-40.

31 Im Vertrag von Amsterdam (1997) wurden u.a. ein Hoher Vertreter etabliert, das Prinzip der konstruktiven Enthaltung und, in Ausnahmefällen, eine qualifizierte Mehrheit eingeführt und die Petersberg-Aufgaben der WEU übernommen.

32 Giegerich, Bastian/Wallace, William: Not Such a Soft Power: the External Deployment of European Forces, in: Survival 46 (2004) $2,166$.

33 Vgl. Giegerich/Wallace (2004), 156-168

34 Vgl. Diedrichs (2012), 27-31. 
to international crises." 35

In der Folge begann mit dem Gipfel von Köln (1999) der Aufbau der Europäischen Sicherheits- und Verteidigungspolitik (ESVP). ${ }^{36}$ Die Gipfel von Helsinki (1999) und Feira (2000) legten durch die Verabschiedung militärischer und nicht-militärischer Headline-Goals weitere Grundlagen. Schliesslich wurden durch den Vertrag von Nizza ein institutioneller Rahmen und durch die Europäische Sicherheitsstrategie A Secure Europe in a Better World auch eine strategische Grundlage für die GSVP geschaffen. Im gleichen Jahr begannen die ersten Missionen. Weitere entscheidende Schritte folgten im Vertrag von Lissabon (2009), in dem die ESVP nun unter der Bezeichnung Gemeinsame Sicherheits- und Verteidigungspolitik (GSVP) verankert ist. Der Vertrag schuf neue institutionelle Strukturen für die GASP/GSVP, vor allem durch die Einführung eines Hohen Vertreters der EU für Aussen- und Sicherheitspolitik und des Europäischen Auswärtigen Dienstes (EEAS). ${ }^{37}$ Zudem wurden die Petersberg-Aufgaben (Art. 43 EUV) ausgeweitet. ${ }^{38}$ Schliesslich sollten die Einführung der Ständigen Strukturierten Zusammenarbeit (SSZ) und des Pooling \& Sharing (S\&P) ${ }^{39}$ helfen, die Fähigkeiten zu verbessern. Abgerundet wurden die Reformen der GSVP durch die Einführung einer Solidaritätsklausel (Art. 222 EUV) für den Fall von Terroranschlägen oder Katastrophen sowie einer Beistandsklausel (Art. 42 Abs. 7 EUV) für den Fall eines bewaffneten Angriffs.

\subsection{Akteure und Entscheidungsprozesse}

Noch stärker als die GASP ist die GSVP intergouvernemental geprägt, das heisst die Mitgliedstaaten sind master of the game: „An den Schaltstellen des Prozesses befinden sich jeweils die Regierungen der Mitgliedstaaten, welche die GSVP sowohl hinsichtlich der politischen und strategischen Grundentscheidungen wie auch mit Blick auf die taktischen und operativen Fragen prägen und formen. " ${ }^{\circ}$

Das Europäische Parlament spielt als supranationale Institution in der GSVP nur eine untergeordnete

35 Franco-British Summit: Joint Declaration on European Defense, Erklärung des britischen Premierministers und des französischen Präsidenten in Saint-Malo, 04.12.1998, URL: http://www. atlanticcommunity.org/Saint-Malo\%20Declaration\%20Text.html (27.01.2014).

36 Vgl. Diedrichs (2012), 27-31.

37 Vgl. Diedrichs (2012), 27-40.

38 Die Petersberg-Aufgaben umfassen gemeinsame Abrüstungsmassnahmen, humanitäre Aufgaben, Rettungseinsätze, Aufgaben der militärischen Beratung und Unterstützung, Aufgaben der Konfliktverhütung und der Erhaltung des Friedens, Kampfeinsätze im Rahmen der Krisenbewältigung, einschliesslich Frieden schaffender Massnahmen und Operationen zur Stabilisierung der Lage nach Konflikten. Ursprünglich wurden die Petersberg-Aufgaben der WEU im Vertrag von Amsterdam von der EU übernommen.

39 Pooling and Sharing bezeichnet die gemeinsame Entwicklung und Beschaffung militärischer Fähigkeiten. Im NATO-Kontext ist dies unter der Bezeichnung Smart Defence bekannt. P\&S lässt sich in drei Kategorien unterteilen: 1) gemeinsame Entwicklung, Produktion und Anschaffung einer Plattform oder eines Systems (z.B. Eurofighter); 2) gemeinsame Nutzung einer militärischen Struktur (z.B. multinationale Einheiten wie die South Eastern European Brigade); 3) Nischenkapazitäten, die eine begrenzte Zahl von Staaten besitzen, die Partnern zur Verfügung gestellt werden. Vgl. Camporini, Vincenzo/Briani, Valerio: L'elemento militare, in: Marrone, Alessandro/Nones, Michele (Hg.): More Europe on Defence or No Europe, Istituto Affari Internazionali, Documenti IAI 13/03, Juni 201 3, 7-9.

40 Diedrichs (2012), 13-14. 
Rolle. ${ }^{41}$ Demgegenüber weisen der Europäische Rat und der Ministerrat ein starkes Gewicht auf. Die Staats- und Regierungschefs im Europäischen Rat legen die Grundlinien der GASP/GSVP fest, das heisst die Richtung, die strategischen Interessen und Ziele. Hierauf aufbauend treffen die Aussenminister im Rat der Europäischen Union Beschlüsse zur Ausgestaltung der GASP/GSVP. ${ }^{42}$ Sind Fragen der GSVP betroffen, können sich anstelle der Aussenminister auch die Verteidigungsminister oder die Aussen- und Verteidigungsminister gemeinsam treffen. Sie fällen Entscheidungen zur GSVP einstimmig. ${ }^{43}$ Geleitet werden die Treffen durch den Hohen Vertreter der EU für Aussen- und Sicherheitspolitik. Dieser ist gleichzeitig Vizepräsident der Europäischen Kommission. Ihm unterstellt ist zudem der EEAS. ${ }^{44}$ Die Arbeit des Rates unterstützen weitere Organisationseinheiten, zum Beispiel der Ausschuss der Ständigen Vertreter (Coreper), das Politische und Sicherheitspolitische Komitee (PSK/COPS) sowie das EUMC (European Union Military Committee) und der Militärstab EUMS (Military Staff of the European Union). Deutlich wird, dass hier ein komplexes Entscheidungsnetzwerk vorliegt, das sowohl Akteure in den Mitgliedstaaten als auch auf europäischer Ebene umfasst. ${ }^{45}$

\subsection{Missionen und Operationen}

Seit 2003 hat die EU über 30 GSVP-Missionen und Operationen lanciert. Sie verfolgt hierbei einen comprehensive approach, der die Existenz von Fähigkeiten sowohl für zivile als auch für militärische Missionen nötig macht. Die Fähigkeiten werden von den Mitgliedstaaten zur Verfügung gestellt. Diese treffen somit zwei Entscheidungen: Zunächst wird auf europäischer Ebene vereinbart, ob eine GSVP-Mission durchgeführt wird. Im Anschluss entscheiden die Mitgliedstaaten, ob sie sich am Einsatz beteiligen. Die bisherigen Missionen und Operationen der GSVP unterscheiden sich teils stark, unter anderem hinsichtlich der Personalgrösse, der Beteiligung der Mitgliedstaaten, der Daver, des Einsatzgebiets, der Kosten und des Umfangs des Mandats. ${ }^{46}$

41 Das Mitentscheidungsverfahren, das seit Lissabon ordentliches Gesetzgebungsverfahren in der EU ist, wird in der GASP/GSVP nicht angewendet. Das Parlament verfügt daher nur über begrenzten Einfluss, vor allem über seine Haushaltsrechte.

42 Vgl. Diedrichs (2012), 46.

43 Für das Krisenmanagement wurde im Vertrag von Lissabon eine sogenannte konstruktive Enthaltung eingeführt. Hierbei kann ein Staat sich bei einer Frage enthalten und ermöglicht somit einen Beschluss. Er darf die beschlossene Politik in der Folge nicht behindern.

44 Vgl. Diedrichs (2012), 48-51.

45 Vgl. Bickerton, Chris J./Irondelle, Bastien/Menon, Anand: Security Co-operation beyond the Nation-State: The EU's Common Security and Defence Policy, in: Journal of Common Market Studies, 49 (201 1) 1, 6.

46 Vgl. Asseburg/Kempin (2009), 164-177. 


\begin{tabular}{|c|c|}
\hline Bezeichnung & Zeit \\
\hline EUPM Bosnia and Herzegovina & 2003-2012 \\
\hline CONCORDIA FYROM & 2003 \\
\hline ARTEMIS DRC & 2003 \\
\hline EUPOL PROXIMA FYROM & 2003-2005 \\
\hline EUJUST Themis Georgia & 2004-2005 \\
\hline EUFOR ALTHEA & seit 2004 \\
\hline EUPOL Kinshasa & $2004-2007$ \\
\hline EUJUST-Lex Iraq & seit 2005 \\
\hline EUSEC DR Congo & seit 2005 \\
\hline EU Support to AMIS Sudan & $2005-2006$ \\
\hline EU BAM Rafah Palestinian Territories & seit 2005 \\
\hline EU BAM Moldova/Ukraine & seit 2005 \\
\hline EUPAT FYROM & $2005-2006$ \\
\hline AMM Banda Aceh & $2005-2006$ \\
\hline EUPOL COPPS Palestinian Territories & seit 2006 \\
\hline EUFOR DRC & 2006 \\
\hline EUPOL DR Congo & seit 2007 \\
\hline EUPOL Afghanistan & seit 2007 \\
\hline EUFOR Chad/C.A.R. & 2008-2009 \\
\hline EUNAVCO/EUNAVOR Atalanta & seit 2008 \\
\hline EUSSR Guinea-Bissau & $2008-2010$ \\
\hline EU MM Georgia & seit 2008 \\
\hline EULEX Kosovo & seit 2008 \\
\hline EUTM Somalia & seit 2010 \\
\hline EUFOR Libya & 2011 \\
\hline EUCAP Sahel/Niger & seit 2012 \\
\hline EUCAP NESTOR Horn of Africa & seit 2012 \\
\hline EU AVSEC South Sudan & seit 2012 \\
\hline EUTM Mali & seit 2013 \\
\hline EUBAM Libya & seit 2013 \\
\hline EUFOR CAR Bangui & seit 2014 \\
\hline
\end{tabular}

Tabelle 1: Zivile und militärische GSVP-Missionen. Eigene Darstellung basierend auf ISIS Europe: CSDP-Map, URL: http://www.csdpmap.eu/mission-chart (27.01.20141, Brüssel; Grevi/Helly/Keohane (2009).

\subsection{Zwischenfazit}

15 Jahre nach dem Gipfel von Saint Malo verfügt die GSVP durch den Vertrag von Lissabon über Instrumente, Prozesse und Institutionen und kann auf Erfahrungen aus einer grösseren Zahl von Missionen zurückgreifen. Gleichzeitig sehen sich die EU und ihre Mitgliedstaaten mit neuen sicherheitspolitischen 
Herausforderungen konfrontiert, die ein gemeinsames Vorgehen als notwendig erscheinen lassen. So ziehen sich die USA zunehmend aus Europa zurück und orientieren sich Richtung Asien/Pazifik, so dass Europa stärker verantwortlich für seine eigene Sicherheit wird. Gleichzeitig treten in der (erweiterten) Nachbarschaft neue Instabilitäten auf. Auch macht die Finanzkrise vor den Verteidigungshaushalten keinen Halt. Einige Mitgliedstaaten haben bereits Probleme, ihre sicherheits- und verteidigungspolitische Handlungsfähigkeit aufrechtzuerhalten. Das komplette Spektrum sicherheitspolitischer Instrumente ist auch für die grösseren Staaten kaum noch zu finanzieren. Schliesslich verändern neve Mächte mit hohen Verteidigungsausgaben die internationale Machtbalance, während gleichzeitig die europäische Rüstungsindustrie droht, durch sinkende Verteidigungshaushalte Innovations- und Wettbewerbstähigkeit zu verlieren. ${ }^{47}$

All dies sind Faktoren, die eine stärkere europäische Zusammenarbeit in Sicherheits- und Verteidigungsfragen begünstigen könnten. Und doch wird die GSVP von Beobachtern in einer "Sinn- und Begründungskrise"48 gesehen. „Bislang haben jedoch weder die institutionellen Neuerungen des Lissabon-Vertrags noch die Finanzkrise zum erhofften Umdenken geführt; vielmehr war zunächst ein Rückzug der GSVP und teilweise eine Renationalisierung der Verteidigungspolitik zu beobachten. "49

\section{Herausforderungen}

Die Auswertung der Think Tank-Publikationen zur GSVP im Umfeld des Dezembergipfels offenbart zahlreiche einzelne Herausforderungen, mit denen die GSVP konfrontiert ist. Verwiesen wird dabei einerseits darauf, dass grundlegende Fragen unbeantwortet sind (Wohin soll sich die GSVP entwickeln? Was sind die strategischen Ziele der GSVP? Wo liegen ihre Prioritäten?). Andererseits werden konkrete Probleme wie das Verhältnis zur NATO, der fragmentierte Rüstungsmarkt, die mangelnde Umsetzung des P\&S oder der Nicht-Einsatz der Battle Groups angesprochen. Im Folgenden erfolgt zunächst eine systematische Analyse der Herausforderungen mit Hilfe des actorness-Ansatzes. Dies erlaubt eine Bewertung der sicherheits- und verteidigungspolitischen Handlungsfähigkeit der EU. Im Anschluss wird nach der Einwirkung nationaler Souveränitäts- und Rollenkonzepte gefragt: Verhindern diese die Ausprägung einer sicherheits-

47 Gründe für eine Stärkung der GSVP analysieren Iso-Markku, Tuomas: European Defence under Scrutiny, The Finnish Institute of International Affairs, FllS Briefing Paper 145, November 2013, 3; Missiroli, Antonio/Gilli, Andrea/Rogers, James: Enabling the Future. European Military Capabilities 2013-2025: Challenges and Avenues, European Union Institute for Security Studies, Report No. 16, May 2013, 16-24; Wientzek, Olaf: Letzte Hoffnung Dezembergipfel? Impulse für die Wiederbelebung der Gemeinsamen Sicherheits- und Verteidigungspolitik, Analysen \& Argumente, 127, Konrad-Adenauer-Stiftung, Berlin, September 2013, 4-5.

48 Von Ondarza, Nicolai/Overhaus, Marco: Die GSVP nach dem Dezember-Gipfel, Stiftung Wissenschaft und Politik, SWP-Aktuell 58, Oktober 2013,4.

49 Wientzek (2013), 3. 
und verteidigungspolitischen actorness der EU?

\subsection{Sicherheits- und verteidigungspolitische actorness der EU}

Die Probleme der GSVP werden zunächst anhand der vier Komponenten der sicherheits- und verteidigungspolitischen actorness (Kohärenz, Autonomie, Autorität und Anerkennung) untersucht.

\section{Kohärenz}

Ein Mangel an Zielkohärenz ist bereits bei grundlegenden Fragen zu erkennen, die in der GSVP nicht oder nur ungenügend geklärt sind. So sind sich die Mitgliedstaaten nicht einig über die Ziele der GSVP, ihre Strategien und Prioritäten. „Denn hier liegt die eigentliche Hürde: Die Staaten der EU haben keine gemeinsame Vorstellung von Sicherheitspolitik."50 Die strategischen Grundlagen der GSVP sind zwar in der Europäischen Sicherheitsstrategie (ESS) aus dem Jahr 2003 niedergelegt, diese ist jedoch in der Zwischenzeit in vielen Punkten überholt und bezieht neuere Entwicklungen wie die zunehmende Instabilität in unmittelbarer und mittelbarer Nachbarschaft der EU nicht ein. ${ }^{51}$ Dieser mangelnde Grundkonsens löst weitere Inkohärenzen aus. So ist auch bei den Missionen und Operationen ein Mangel an Zielkohärenz zu diagnostizieren. Die grosse Zahl an Missionen und Erfolge, die teils zu verzeichnen sind, verschleiern, dass kein Konsens darüber besteht, wann GSVP-Missionen lanciert und wie diese ausgestaltet werden sollen: „Während Konsens darüber besteht, dass die EU weiterhin zivile und militärische Instrumente für das Krisenmanagement vorhalten will, gibt es vor allem bei militärischen Operationen keine Einigkeit über das Wann, Wie und Wo." 52

In der Folge erscheint die Auswahl der Missionen willkürlich. So begannen ab 2009 fast drei Jahre kaum neve Missionen, während seit 2012 sechs neve GSVP-Missionen lanciert wurden. Einige Missionen gehen auf den Wunsch eines einzelnen Staates zurück, viele, zum Beispiel die Mission im Südsudan, verfügen nur über wenige Einsatzkräfte und können in die Rubrik "Symbolpolitik"53 eingeordnet bzw. als reiner "Existenznachweis der europäischen Sicherheitspolitik"54 gewertet werden. Auch dauert es teils sehr lange, bis ein Beschluss getroffen wird und die Missionen schliesslich einsatzbereit sind: „Die EU ist

\footnotetext{
50 Winter (2013), 7
}

51 Vgl. Iso-Markku (2013), 4. Überarbeitet wurde die ESS 2008.

52 Linnenkamp, Hilmar/Mölling, Christian: Eine Agenda für den Europäischen Verteidigungsrat 2013, Stiffung Wissenschaft und Politik, SWP-Aktuell 57, Oktober 2013, 2. Ähnlich Iso-Markku (2013), 4: „One of the EU's main problems in the area of security and defence is often considered to be the lack of strategy. The argument is that without clearly defined strategic priorities, the member states will never be able to agree on how, when and where to act, what to aim at in their operations and what kind of capabilities to develop in the long run."

53 Von Ondarza/Overhaus (2013), 1.

54 AK Internationale Sicherheitspolitik (2012), 3. Hier wird davon ausgegangen, dass die Missionen Signale nach innen, an die NATO und an die USA sind. 
derzeit entweder nicht willens oder fähig, einen raschen Beschluss zur Entsendung einer GSVP-Mission zu fassen. Die lange Anlaufzeit für die Trainingsmission in Mali ist nur ein Beispiel dafür." ${ }^{\text {"55 }}$

Besondere Kritik in jüngerer Zeit löste das Vorgehen der Europäer in Libyen aus. Zunächst wiesen die EU-Mitgliedstaaten bei der Abstimmung zur Libyen-Resolution im UN-Sicherheitsrat kein einheitliches Abstimmungsverhalten auf. ${ }^{56} \mathrm{Im}$ Anschluss intervenierten Frankreich und Grossbritannien alleine. Die später vorgesehene militärische GSVP-Mission EUFOR Libya schliesslich entwickelte sich zu einer "ghost CSDP operation"57, die nur auf dem Papier bestand und in der Praxis nie realisiert wurde: „EUFOR Libya's failure severely affected the EU's image as an international security actor [...]." 58 Erst im Mai 2013 wurde mit EUBAM Libya eine zivile Mission beschlossen.

Schliesslich wird fehlende Zielkohärenz auch bei der Beschaffung von Fähigkeiten und im Umgang mit der Rüstungsindustrie deutlich. Bei der Beschaffung von Fähigkeiten mangelt es weiterhin an Abstimmung, so dass einerseits deutliche Fähigkeitslücken bestehen und nicht geschlossen werden, während an anderer Stelle Überfluss herrscht. Die unkoordinierten Einsparungen in den Verteidigungshaushalten im Zuge der Finanzkrise verschärfen diese Situation. So schrumpften die Verteidigungshaushalte der EU-Staaten zusammengenommen zwischen 2008 und 2013 von 200 Milliarden Euro auf 170 Milliarden Euro.59 Auch im Umgang mit der Rüstungsindustrie weisen die Mitgliedstaaten keine kohärenten Ziele auf. In einem zersplitterten Markt produzieren europäische Firmen teils ähnliche Güter und konkurrieren um Aufträge aus dem Ausland, während economies of scale ungenutzt bleiben. Auch die Interoperabilität der Fähigkeiten der Mitgliedstaaten ist nicht gewährleistet. Trotzdem wehren sich diese gegen eine Stärkung des Binnenmarktes für Rüstungsgüter und kaufen in der Regel bei Anbietern aus dem eigenen Land oder

55 Vgl. Wientzek (2013), 4. Vgl. auch Heyer, Julia Amalia/Puhl, Jan/Repinski, Gordon/Schult, Christoph: Rechnung fürs Nichtstun, in: Der Spiegel $51 / 2013,86$.

56 Die Resolution 1973 des UN-Sicherheitsrates sah u.a. die Einrichtung einer Flugverbotszone und die Ergreifung aller notwendigen Massnahmen zum Schutz der Bevölkerung vor. Deutschland enthielt sich, u.a. gemeinsam mit Russland und China, während die EU-Partner Grossbritannien, Frankreich und Portugal sowie die USA der Resolution zustimmten. Begründet wurde die Enthaltung damit, dass sich die Bundesrepublik nicht an militärischen Massnahmen beteiligen wolle.

57 Hatzigeorgopoulos, Myrto/Fara-Andrianarijaona, Lorène: EUBAM Libya: Story of a Long-awaited CSDP Mission, isis Europe, European Security Review 66, Mai 2013, 3.

58 Hatzigeorgopoulos (2013), 2. Der Beginn von EUFOR Lybia wurde von einer Anfrage des UN Office for Co-ordination of Humanitarian Affairs (OCHA) abhängig gemacht. Die OCHA verfügte jedoch bereits über die Mittel, die die EU anbot, und benötigte keine militärischen Mittel. Daher traf eine solche Anfrage nicht ein. Gomes fragt vor diesem Hintergrund, ob die am 1. April beschlossene Mission ein Aprilscherz gewesen sei. Vgl. Gomes, Ana: Was Eufor Libya an April fool's joke?, in: EUobserver, 13.07.20 1 1, URL: http:// euobserver.com/opinion/32624 (28.01.2014). Am 22. Mai 2013 beschloss der Rat eine neve zivile GSVP-Mission in Libyen, EUBAM Libya, die bei der Verbesserung des Grenzmanagements helfen soll und dabei Ausbildung und Beratung für die libyschen Behörden leistet. Eine ausführliche Darstellung von EUBAM Libya und ihrer Vorgeschichte bietet Hatzigeorgopoulos (2013).

59 Die Verteidigungshaushalte in Finnland und Dänemark blieben stabil; diejenigen von Polen und Schweden sind gewachsen. In den meisten mittelgrossen Staaten sind die Ausgaben um 10 bis 15\% gesunken, in einigen kleineren wie Lettland und Litauen gar um über $20 \%$. Vgl. O'Donnell, Clara Marina: The Trials and Tribulations of European Defence Co-operation, Centre for European Reform, July 2013. 
direkt ausserhalb der EU. 60

"Winkt irgendwo auf der Welt ein Großauftrag, dann ziehen die europäischen Nationen gegeneinander ins Feld. Wollen sie hingegen selbst militärische Ausrüstung anschaffen, schotten sie sich ab, schalten alle Regeln der Vernunft und des Marktes aus, oft genug, um die heimische Rüstungsindustrie zu schützen. Zu viel Wettbewerb nach außen, kein funktionierender Wettbewerb innerhalb der EU, so betreiben die nationalen Regierungen in der Praxis das, was sie auf dem Papier eine ,Gemeinsame Sicherheits- und Verteidigungspolitik' nennen. "61

Versuche der Europäischen Kommission, die Mitgliedstaaten zur Öffnung der nationalen Rüstungsmärkte zu animieren, sind kaum von Erfolg gekrönt, da diese ihre eigenen Rüstungsfirmen schützen wollen. Deutlich wurde die Bedeutung, die der eigenen Rüstungsindustrie beigemessen wird, unter anderem beim gescheiterten Zusammenschluss von EADS und BAE Systems. ${ }^{62}$

Obwohl die taktische Kohärenz, das heisst die Bereitschaft zur Kompromissfindung, zum bargaining oder zu Kompensationslösungen im Gegensatz zur Zielkohärenz ausgeprägt erscheint, treten auch hier Probleme auf. Als Beispiel lässt sich der Umgang mit den Battle Groups ${ }^{63}$ anführen, die bis heute nicht eingesetzł wurden. Die Staaten, die die Battle Groups stellen, müssten einem Einsatz zustimmen. In der Realität ist die Bereitschaft hierzu gering. Stattdessen werden Ersatzlösungen gesucht und andere Einsatzkräfte genutzt, die jene Staaten bereitstellen, die sich an der entsprechenden Mission beteiligen wollen. So gingen Beobachter zum Beispiel für die Missionen im Kongo und in Mali von einem Mehrwert durch einen Einsatz der Battle Groups aus. Ein solcher wurde aber für Mali durch die Mitgliedstaaten nicht diskutiert und für den Kongo durch die Staaten, die die Battle Groups zu diesem Zeitpunkt stellten, abgelehnt. ${ }^{64}$ Winter kommt daher zu dem Fazit, dass sich "[...] offensichtlich immer irgendein Grund findet, die ,Battle Group' nicht einzusetzen [...]",65 und Iso-Markku stellt fest: „[...] [T]he battlegroups

60 Vgl. Iso-Markku (20 13), 7-8. Als Beispiel lässt sich die Existenz dreier europäischer Kampfjet-Programme anführen (Rafale, Eurofighter, Gripen), die die Interoperabilität behindern, Einsparungen verhindern und miteinander im Export konkurrieren. Vgl. O'Donnell (2013a).

61 Repinski, Gordon/Schult, Christoph/Traufetter, Gerald: Brennende Unterhosen, in: Der Spiegel, 50/2013, 30.

62 Vgl. O’Donnell (2013), 5; Deckstein, Dinah/Traufetter, Gerald: Feld voller Feinde, in: Der Spiegel, 41/2012, 82-83. Vgl. zur Position der Kommission zum Markt im Sicherheits- und Verteidigungssektor Maulny, Jean-Pierre: European Commission Communication on the Defence and Security Sector: Towards a Definition of a Strategic EDTIB? , Institut de Relations Internationales et Stratégiques, Les Notes de L'ris, September 2013 ; Fiott, Daniel: More Competitive, More Efficient? The 2013 European Commission Defence Communication, EGMONT Royal Institute for International Relations, Security Policy Brief, No. 49, September 2013.

63 Die EU Battle Groups sind Krisenreaktionskräfte, die innerhalb von zehn Tagen einsatzbereit sind. Es existieren parallel zwei in der Regel multinationale Battle Groups, die von den Mitgliedstaaten für sechs Monate bereitgestellt werden.

64 Vgl. Wientzek (2013), 3.

65 Winter (2013), 7. 
have become a symbol of the EU's unwillingness to deploy militarily."

Insgesamt zeigt sich, dass ein Grundkonsens zur GSVP fehlt und es auch bei einzelnen Fragen an einem gemeinsamen Vorgehen der Mitgliedstaaten mangelt. Die Verwirklichung von Kohärenz, einem Kern der sicherheits- und verteidigungspolitischen Handlungsfähigkeit, stellt somit weiterhin eine Herausforderung für die GSVP dar.

\section{Autorität}

Durch den Vertrag von Nizza (2001) wurde die GSVP offiziell in den Verträgen verankert, durch den Lissabonner Vertrag wurden die Bestimmungen zur GSVP erheblich ausgeweitet. Die vertraglichen Grundlagen sind somit vorhanden. Allerdings fällt auf, dass wichtige Bestimmungen aus dem Vertrag von Lissabon kaum oder überhaupt nicht genutzł werden. Zu nennen sind insbesondere die SSZ, die Battle Groups und die Solidaritäts- und Bündnisklauseln. Auffällig isł zudem, dass die Nutzung der GSVP in der Praxis für die Mitgliedstaaten keine Selbstverständlichkeit ist. Diese zögern teils, auf die GSVP zuzugreifen und bevorzugen andere Formen der Kooperation: „Weder im Falle Libyens noch Malis wurde die Möglichkeit eines (militärischen) GSVP-Einsatzes ernsthaft diskutiert." ${ }^{67}$ Insbesondere bei verteidigungspolitischen Themen fungiert die GSVP nicht zwangsläufig als erste Anlaufadresse. ${ }^{68}$ Somit bestehen Probleme, die Autorität in der Praxis umzusetzen. Dies kann sich negativ auf die Handlungsfähigkeit der GSVP auswirken.

\section{Autonomie}

Autonomie umfasst institutionelle Unterscheidbarkeit und Unabhängigkeit. Eine institutionelle Unterscheidbarkeit für die GASP/GSVP besteht formal vor allem durch die im Lissabonner Vertrag verankerten institutionellen Reformen. ${ }^{69}$ Diese führten den Hohen Vertreter für die Aussen- und Sicherheitspolitik und den EEAS ein und verankerten die Europäische Verteidigungsagentur (EDA) in den Verträgen. Darüber hinaus existieren weitere GASP- oder GSVP-spezifische Organisationseinheiten wie der Militärstab. Allerdings bleiben die Institutionen teilweise schwach:

"In order to remedy some of these shortcomings, the member states agreed in the Lisbon Treaty to establish the European External Action Service (EEAS) as the new hub of the EU's external

\footnotetext{
66 Iso-Markku (2013), 5.

67 Wientzek (2013), 4.

68 Wientzek weist darauf hin, dass sich mit dem Europäischen Parlament ausgerechnet eine kaum eingebundene Institution zum grössten Befürworter der GSVP entwickelt hat. Vgl. Wientzek (2013), 4.

69 Vgl. Bonvicini/Faleg (2013), 1 1-15.
} 
activities and expand the competences of the High Representative. However, the impact of these institutional adjustments on the effectiveness of the EU's external action is still being debated. The CSDP, above all, has seemingly lost out in the institutional context."70

Bonvicini/Faleg diagnostizieren hiermit einhergehend ein leadership-Problem, das sie nicht nur auf ein geringes Engagement der Mitgliedstaaten, sondern auch auf die mangelnde Weiterentwicklung des Politikfelds durch die Hohe Vertreterin sowie auf interinstitutionelle Konflikte zwischen Rat und Kommission zurückführen. ${ }^{71}$ Besonders skeptisch gesehen wird darüber hinaus die Lage der Verteidigungsagentur EDA:

„Idealtypisch ist die EDA also die zentrale Koordinierungsstelle für alle Pooling and Sharing-Maßnahmen. In der politischen Realität hat sie diese Rolle bisher noch nicht ausfüllen können. Zu stark waren die nationalen Vorbehalte, die Entscheidungs- und Koordinationsautorität aus der Hand zu geben. Zu schwach war aber auch der politische Wille der EDA-Führung, den ihr gegebenen Handlungsspielraum auszureizen."72

Kritisiert werden fehlende finanzielle Ressourcen, aber auch die Auswirkungen der Ko-Existenz von EDA und Europäischer Kommission. Mit dem Binnenmarkt für Rüstungsgüter berührt ein für die EDA wichtiges Feld den Aufgabenbereich der Kommission, die der EDA teils bezüglich Personal, Ressourcen und Knowhow überlegen ist. ${ }^{73}$ Darüber, welche Rolle die supranationale Kommission in Zukunft übernehmen soll, besteht keine Einigkeit zwischen den Mitgliedstaaten. ${ }^{74}$

Neben der institutionellen Unterscheidbarkeit fragen Jupille/Caporaso nach der Unabhängigkeit: Die EU soll mehr als die Summe ihrer Teile sein, nach eigenem Ermessen Ziele festlegen, entscheiden und implementieren. Da die GSVP stark intergouvernemental geprägt ist, spielen in diesem Politikfeld jedoch die Mitgliedstaaten eine wichtige Rolle bei der Definition der Interessen, der Entscheidungsfindung und der Implementierung. Gleichzeitig können die Mitgliedstaaten im Rahmen der GSVP gemeinsam mehr erreichen als ein einzelner Staat. So könnten auch die grösseren Mitgliedstaaten die Missionen kaum alleine stemmen.

Zu beachten ist aber, dass 22 der 28 EU-Staaten Mitglieder der NATO sind. Daher stellt sich die Frage,

70 Iso-Markku (2013), 4.

71 Vgl. Bonvicini, Gianni/Faleg, Giovanni: L'elemento istituzionale, in: Marrone, Alessandro/Nones, Michele (Hg.): More Europe on Defence or No Europe, Istituto Affari Internazionali, Documenti IAI 13/03, Juni 2013, 11-15.

72 Keller et al (2012), 87.

73 Vgl. Linnenkamp/Mölling (2013), 3.

74 Vgl. Iso-Markku (2013), 7. Die Kommission wird aktuell immer stärker in den Bereich Fähigkeiten einbezogen. Als Hüterin des Binnenmarktes spielt sie zunehmend eine Rolle im europäischen Rüstungsmarkt. Sie will bei der Unterstützung von dual-use capabilities mitwirken. Allerdings steht u.a. Grossbritannien einer Stärkung der Kommission in der Sicherheits- und Verteidigungspolitik skeptisch gegenüber. 
ob die GSVP auch gegenüber der NATO einen Mehrwert aufweist. Einerseits wird skeptisch gesehen, dass das Nebeneinander von NATO und GSVP zu Duplizierungen führen könnte, zum Beispiel bei der Schaffung eigener permanenter ziviler und militärischer Kommandostrukturen der GSVP. Auch die neue Beistandsklausel scheint angesichts der existierenden NATO-Bündnispflicht wenig Mehrwert zu erbringen. Initiativen wie P\&S und die Battle Groups weisen ebenso Pendants auf NATO-Ebene auf (Smart Defence und NATO Response Force). Schliesslich ist die GSVP weiterhin kaum in der Lage, grössere Missionen ohne US-amerikanische Hilfe durchzuführen. Andererseits wird in diesem Zusammenhang häufig auf den comprehensive approach der EU verwiesen: Im Gegensatz zur NATO verfügt die EU über das gesamte Spektrum militärischer und ziviler, humanitärer und entwicklungspolitischer Instrumente. ${ }^{75}$ Hier ist also ein Mehrwert vorhanden.

Abschliessend ist nach den Ressourcen zu fragen, die Harnisch/Stahl neben der Unabhängigkeit und der Unterscheidbarkeit als dritten Bestandteil der Autonomie identifizieren. Hingewiesen wurde oben bereits auf die problematische finanzielle Ausstattung der EDA. Bedeutender ist jedoch, dass die GSVP auch bei Missionen und Operationen auf die Ressourcen der Mitgliedstaaten angewiesen ist, auf die sie jedoch nicht immer schnell und unkompliziert zugreifen kann:

"Gleich, ob ziviler oder militärischer Natur, GSVP-Missionen haben Schwierigkeiten, das benötigte Personal aus den Mitgliedstaaten zu erhalten. Die grundsätzliche Zurückhaltung der Mitgliedstaaten, sich in GSVP-Missionen zu engagieren, liegt wohl auch in der Finanzierungsmethode begründet. So wird der Großteil der Kosten einer Mission nach dem Prinzip ,Costs lie where they fall' finanziert, wonach der Einsatz weitgehend (im Schnitt zu etwa 90\%) von den engagierten Mitgliedstaaten zu tragen ist. Lediglich rund 10 Prozent der Kosten werden gemeinsam über den sogenannten ,Athena-Mechanismus' finanziert." ${ }^{\text {"76 }}$

Zusammengefasst weist die GSVP einerseits eine formelle institutionelle Unterscheidbarkeit und andererseits zumindest Züge von Unabhängigkeit auf. Gleichzeitig lassen sich in den Institutionen weiterhin Probleme erkennen, und auch die Koexistenz mit der NATO ist als Herausforderung für die GSVP anzusehen, etwa bezüglich des Zugriffs auf die für die Missionen und Operationen benötigten Ressourcen der Mitgliedstaaten.

75 Vgl. Wientzek (2013), 4-5.

76 Wientzek (2013), 4. Ausgaben mit sicherheits- und verteidigungspolitischen Bezügen werden von den Mitgliedstaaten selbst getragen (costs lie where they fall). Nur Kosten, die nicht einem Mitgliedstaat zugeordnet werden können (common costs), werden über den Athena-Mechanismus gemeinsam getragen. Vgl. Diedrichs (2012), 84-92. 


\section{Anerkennung}

Seit Inkrafttreten des Vertrags von Lissabon verfügt die EU über eine eigene Rechtspersönlichkeit (Art. 47 EUV). ${ }^{77}$ Sie kann somit internationale Abkommen abschliessen und internationalen Organisationen und Vertragswerken beitreten. Wichtiger für die sicherheits- und verteidigungspolitische Handlungsfähigkeit ist aber die Anerkennung in Form praktischer Zusammenarbeit. Hier stösst die GSVP weiterhin auf Probleme, vor allem in der Kooperation mit der NATO. Das Verteidigungsbündnis ist für die meisten Mitgliedstaaten der EU der wichtigste Sicherheits- und Verteidigungsakteur. Hinterfragt wird daher weiterhin, ob NATO und GSVP tatsächlich komplementär sind oder doch Konkurrenten. ${ }^{78}$

Belastet wird das Verhältnis zwischen beiden Organisationen durch die Zypernfrage. Seit dem Beitritt des Landes zur EU 2004 ist eine "Eiszeit"79 ausgebrochen, das heisst es bestehen kaum mehr offizielle Kooperationen. ${ }^{80}$ Einzig informelle Gespräche sind möglich und finden nicht nur auf Arbeitsebene, sondern auch zwischen dem Hohen Vertreter der EU und dem Generalsekretär der NATO statt. Gleichzeitig teilen beide Organisationen aber auch grundlegende Ziele und Herausforderungen. "NATO is formed of many of the same Member States, and is thus impaired by the same problems: a lack of funding, a lack of joint responsibilities, a lack of joint vision, a lack of common will and multiplying threats. National disputes impede work both within and between these organisations." ${ }^{81}$

Ohne die Kooperation mit der NATO fehlen der EU unter anderem wichtige Planungs- und Kommandofähigkeiten sowie Ausrüstung. Ursprünglich sollte hier das Berlin-Plus-Abkommen Lösungen bieten, das jedoch auf Grund der türkisch-griechisch-zypriotischen Blockade nur im Rahmen der Operation Althea Anwendung findet. Der blockierte Zugriff der EU auf das NATO-Hauptquartier und der fehlende Informationsaustausch erschweren das Alltagsgeschehen und sorgen für Langsamkeit und Ineffizienz. Existieren trotz der Blockaden in der Praxis Kooperationen, zum Beispiel zwischen den Operationen Ocean Shield und Atalanta, werden diese zwar in der Regel positiv beurteilt, gleichzeitig scheinen sich NATO und EU aber mit der ungelösten Situation abgefunden zu haben: "It seems that Brussels and the Member States

77 Zuvor existierte eine Säulenstruktur: Die zweite Säule umfasste die GASP, die dritte Säule die polizeiliche und justizielle Zusammenarbeit in Strafsachen und die erste Säule die vergemeinschafteten weiteren Politikfelder. Nur die Europäische Gemeinschaft und die Europäische Atomgemeinschaft verfügten über eine Rechtspersönlichkeit.

78 Auch in der US-amerikanischen Politik wird dies offiziell seit 2008 nicht mehr diskutiert. Allerdings wird ein Widerspruch in konservativen Kreisen weiterhin als gegeben angesehen. So kritisiert Coffey die GSVP scharf und sieht sie im Widerspruch zu US-amerikanischen Interessen. Er sieht keine Vorteile in einer Stärkung der GSVP, die er als "Papiertiger" bezeichnet. Vgl. Coffey, Luke: EU Defense Integration: Undermining NATO, Transatlantic Relations, and Europe's Security, The Heritage Foundation, Backgrounder, No. 2806, 6.6.2013, 7.

79 Hopia, Henna: Breaking Down the Walls: Improving EU-NATO Relations, Centre for European Studies 2013, 9.

80 Die Republik Zypern ist Mitglied der EU, die Türkei Mitglied der NATO. Die bilateralen Streitigkeiten zwischen beiden Staaten um den Nordteil der Insel Zypern, den die Türkei 1974 militärisch besetzte, behindern die Zusammenarbeit beider Organisationen.

81 Hopia (2013), 8. 
have given up trying and have accepted this as an immutable reality. ${ }^{182}$

Deutlich wird, dass die GSVP in allen vier Komponenten, Kohärenz, Autonomie, Anerkennung und Autorität, auf Herausforderungen stösst. Dabei haben vor allem die Probleme, die sich in einer mangelnden Kohärenz verorten lassen, teils grundsätzlichen Charakter. Probleme der Autorität sind partiell auf Faktoren wie die Zypernfrage zurückzuführen, die alleine durch die EU und ihre Mitgliedstaaten kaum gelöst werden können. Auffällig ist schliesslich, dass in den Kategorien Kohärenz, Autonomie und Autorität viele Herausforderungen ihre Ursache auf nationaler Ebene finden. Daher wird im Folgenden auf die Bedeutung von Souveränitäts- und Rollenkonzepten der Mitgliedstaaten eingegangen.

\subsection{Souveränitätskonzepte der Mitgliedstaaten}

Obwohl der Sicherheits- und Verteidigungspolitik in vielen Mitgliedstaaten ein eher geringer Stellenwert beigemessen wird, ${ }^{83}$ ist sie ein Politikfeld mit besonderer Bedeutung, berührt sie doch den Kern nationaler Souveränität: „Defence policy remains a bastion of national sovereignty, and this makes cooperation difficult to achieve." ${ }^{44}$ Abgabe von Souveränität stellt für die Mitgliedstaaten in diesem Bereich daher eine besondere Schwierigkeit dar. Betrachtet man eine Zusammenstellung der oben identifizierten Herausforderungen und die Frage, ob hier Probleme mit der Souveränitätsabgabe auftreten, so ergibt sich folgendes Bild:

\begin{tabular}{|c|c|}
\hline Herausforderung & Abgabe von Souveränität \\
\hline \multicolumn{2}{|l|}{ Kohärenz } \\
\hline $\begin{array}{l}\text { Fehlender Grundkonsens zur } \\
\text { GSVP }\end{array}$ & $\begin{array}{l}\text { Es fehlen gemeinsame Ziele, Strategien und Prioritäten. Diese würden } \\
\text { eine Entscheidung darüber voraussetzen, inwieweit die Mitgliedstaaten } \\
\text { bereit sind, Souveränität abzugeben. }\end{array}$ \\
\hline Missionen & $\begin{array}{l}\text { Es fehlt ein gemeinsamer Ansatz, wann und mit welchen Zielen Missi- } \\
\text { onen lanciert werden. Jedes Land entscheidet souverän darüber, ob es } \\
\text { einer Mission zustimmt und ob es sich beteiligt. }\end{array}$ \\
\hline Beschaffung von Fähigkeiten & $\begin{array}{l}\text { Eine Koordination auf europäischer Ebene könnte helfen, Duplizierungen } \\
\text { zu vermeiden und Fähigkeitslücken zu schliessen. Die Staaten sind aber } \\
\text { nur in geringem Masse bereit, hier Souveränität an die EU abzugeben. }\end{array}$ \\
\hline
\end{tabular}

82 Hopia (2013), 31. Vgl. zu den EU-NATO-Beziehungen auch Flockhart, Trine: 'Me Tarzan - You Jane': The EU and NATO and the Reversal of Roles, in: Perspectives on European Politics and Society 12 (201 1) 3, 263-282.

83 Vgl. von Ondarza/Overhaus (2013), 1.

84 Gross, Eva/Menon, Anand: European Defence: An Inventory for the December Summit, in: dies. (Hg.): CSDP Between Internal Constraints and External Challenges, EU Institute for Security Studies, Report No. 17, Oktober 2013, 7. 


\begin{tabular}{|c|c|}
\hline Rüstungsmarkt & $\begin{array}{l}\text { Ein europäischer Rüstungsmarkt könnte die Interoperabilität stärken und } \\
\text { helfen, economies of scale zu nutzen. Die Mitgliedstaaten sehen in eige- } \\
\text { ner Rüstungsindustrie aber ein Zeichen von Unabhängigkeit und wollen } \\
\text { nationale Firmen und Arbeitsplätze schützen. }\end{array}$ \\
\hline Battle Groups & $\begin{array}{l}\text { Die europäischen Battle Groups können nur dann eingesetzł werden, } \\
\text { wenn die Staaten, die die Einsatzkräfte stellen, zustimmen. }\end{array}$ \\
\hline \multicolumn{2}{|l|}{ Autorität } \\
\hline $\begin{array}{l}\text { Nicht-Umsetzung von Bestim- } \\
\text { mungen }\end{array}$ & $\begin{array}{l}\text { Aspekte, die die nationale Souveränität stark berühren, vor allem die } \\
\text { SSZ und die Battle Groups, werden nicht oder nur unzureichend umge- } \\
\text { setzt. }\end{array}$ \\
\hline GSVP als zweite Wahl & $\begin{array}{l}\text { Die Mitgliedstaaten bevorzugen häufig Kooperationsformen, in denen } \\
\text { sie in stärkerem Masse Souveränität bewahren können (z.B. bilaterale } \\
\text { Kooperationen). }\end{array}$ \\
\hline \multicolumn{2}{|l|}{ Autonomie } \\
\hline EDA & $\begin{array}{l}\text { Die EDA bleibt schwach, u.a. weil die Mitgliedstaaten die Koordination } \\
\text { nicht aus der Hand geben und weiter souveräne Entscheidungen treffen } \\
\text { wollen. }\end{array}$ \\
\hline Finanzen & $\begin{array}{l}\text { Die GSVP bleibt auf die finanziellen Mittel der Mitgliedstaaten und die } \\
\text { Bereitstellung von Personal angewiesen. Die Staaten agieren hier teils } \\
\text { zurückhaltend. }\end{array}$ \\
\hline
\end{tabular}

Tabelle 2: Herausforderungen, die auf Problemen durch Souveränitätsabgabe basieren (eigene Darstellung).

In drei der vier Komponenten existieren somit Herausforderungen, die direkt oder indirekt mit dem Wunsch der Mitgliedstaaten, ihre Souveränität zu behalten, kollidieren. Hinzu kommen Herausforderungen auf europäischer Ebene (unter anderem Probleme innerhalb der Institutionen, interinstitutionelle Konflikte) und im Verhältnis zur NATO.

Beispielhaft lassen sich die hieraus entstehenden Konsequenzen anhand des P\&S zeigen. Wie oben festgestellt, weisen die Mitgliedstaaten deutliche Fähigkeitslücken auf, beispielsweise im Lufttransport. ${ }^{85}$ Gleichzeitig duplizieren sie Ausstattung und Training. In der Folge existieren neben Fähigkeitslücken auch Bereiche, in denen Überfluss herrscht: „Countries are sustaining ,overcapacities' but at the same time failing to invest in critical shortfalls." 86 Es wird jedoch für den einzelnen Staat immer schwieriger, alle

85 Vgl. Iso-Markku (2013), 6; Hopia (2013), 19; Wientzek (2013), 4. In Libyen waren Frankreich und Grossbritannien innerhalb kurzer Zeit auf US-amerikanische Unterstützung angewiesen. Vgl. Camporini/Briani (2013), 7-9.

86 Hopia (2013), 17. 
benötigten Fähigkeiten zu entwickeln und zu besitzen. ${ }^{87}$ Die Finanzierung ist selbst für grössere Staaten kaum zu stemmen und wird durch das Schrumpfen der Verteidigungshaushalte im Zuge der Finanzkrise zusätzlich erschwert. Hier könnte das P\&S helfen. Tatsächlich werden in der Literatur teils erste Fortschritte im P\&S unter dem Eindruck der Finanzkrise diagnostiziert. So wurden 2011 elf P\&S-Projekte bei der EDA angemeldet, 2012 weitere sieben (unter anderem zur Luftbetankung). Gleichzeitig ist ein mangelnder Wille für ein weiteres gemeinsames Vorgehen festzustellen. ${ }^{88}$ Als grösste Schwierigkeit erweisen sich Souveränitätsvorbehalte: „Die Fortschritte sowohl bei Pooling und Sharing als auch bei Smart Defence sind bisher enttäuschend. Als größtes Hindernis für eine engere militärische Kooperation haben sich die Vorbehalte gegenüber der Abgabe nationaler Souveränität gezeigt. "89

Statt stärker miteinander zu kooperieren, ziehen es die Staaten vor, über kleine und kaum einsetzbare „Bonsai-Armeen"90 zu verfügen. Die Bereitschaft, in diesem sensiblen Bereich Souveränität abzugeben, miteinander zu kooperieren und so Zugriff auf die Fähigkeiten der Partner zu erhalten, ist gering. Dies wurzelt auch in Bedenken hinsichtlich der Verlässlichkeit der Partner:

"Countries remain wary of relying on others for military capabilities. States fear that shared military forces may become unavailable if a partner disapproves of a particular operation. For many NATO members, such concerns were exacerbated when Germany withdrew its pilots from the alliance's jointly owned surveillance aircraft (AWACS) during the deployment to Libya - even though Berlin sent more AWACS pilots to Afghanistan in order to free up military personnel from other NATO countries for the Libya operation." ${ }^{91}$

In der Konsequenz agieren die Staaten nicht nur beim P\&S äusserst zurückhaltend, sondern auch bei der Abstimmung von Einsparungen in den Verteidigungshaushalten. ${ }^{92}$ Dies, obwohl aufeinander abgestimmte Kürzungen das Schrumpfen der Verteidigungshaushalte abfedern könnten.93

Zusammenfassend bewegen sich die Mitgliedstaaten in der GSVP in einem Spannungsfeld: Einerseits sehen sich die Staaten wachsendem finanziellen Druck ausgesetzt. Alleine können sie die benötigten Fä-

87 Vgl. Iso-Markku (2013), 6.

88 Vgl. Hopia (2013), 19; Zarandi, Maik: Europäische Insellösungen als Fundament einer Stärkung der europäischen Verteidigungsfähigkeiten?, Analysen \& Argumente, 130, Konrad-Adenaver-Stiftung, Berlin, Oktober 2013, 4.

89 Zarandi (2013), 3. Ähnlich Keller et al (2012), 84: „Die Bilanz der bisherigen europäischen Pooling and Sharing-Anstrengungen ist ernüchternd. Die Furcht vor einem nationalen Souveränitätsverlust war bisher stets das größte Hindernis für ein erfolgreiches Pooling and Sharing."

90 Missiroli et al (2013), 12.

91 O'Donnell (2013), 2.

92 Vgl. Wientzek (2013), 4.

93 Vgl. Fiott (2013), 4. 
higkeiten kaum mehr finanzieren. Andererseits ist es für Staaten sehr schwierig, in einem solch sensiblen Bereich wie der GSVP Souveränität abzugeben und gemeinsam zu agieren. Neben der nationalen Souveränität wirken hier die aussenpolitischen Rollenkonzepte der Mitgliedstaaten ein, die zudem Auslöser für Glaubwürdigkeits- und Vertrauensprobleme sind.

\subsection{Rollenkonzepte der Mitgliedstaaten}

In der GSVP treffen 28 Staaten mit teils stark variierenden aussenpolitischen Rollenkonzepten aufeinander. Diese können Auslöser dafür sein, dass die Staaten in der GSVP verschiedene Ziele, Strategien und Grundkonzepte befürworten, unterschiedliche Instrumente bevorzugen und bei der Planung von Missionen und Operationen nicht die gleichen Interessen verfolgen. Auch können sie zu Missverständnissen zwischen den Staaten führen. Bereits die aussenpolitischen Rollenkonzepte der ,Grossen Drei', der Zivilmacht Deutschland und der Militärmächte Frankreich und Grossbritannien, sind teils nicht miteinander in Einklang zu bringen. Auch viele kleinere Staaten weisen deutlich voneinander abweichende Rollenkonzepte auf. Zurückzuführen ist dies unter anderem auf folgende Aspekte:94

\begin{tabular}{|l|l|}
\hline Ursachen & Beispiele \\
\hline Historische Erfahrungen & $\begin{array}{l}\text { Einige Staaten, z.B. Grossbritannien, Frankreich und die Niederlande, sind } \\
\text { ehemalige Kolonialmächte, manche haben Erfahrungen mit Diktaturen ge- } \\
\text { macht, andere befanden sich im Einflussbereich der UdSSR. }\end{array}$ \\
\hline $\begin{array}{l}\text { Quantitativ grosse Staaten wie Deutschland stossen auf kleine Länder wie } \\
\text { Malta und Luxemburg. Die Grösse beeinflusst u.a. die Bedeutung von Aus- } \\
\text { senpolitik und die Interessen der Staaten. }\end{array}$ \\
\hline Leitlinien und Mittel & $\begin{array}{l}\text { Die Mehrzahl der EU-Staaten ist auch Mitglied der NATO, andere sind } \\
\text { neutral. Traditionelle Militärmächte und Atommächte stossen auf Staaten, die } \\
\text { Militäreinsätzen skeptisch gegenüberstehen. } \\
\text { Überzeugte Transatlantiker treffen auf Europäer und auf Staaten, die zwi- } \\
\text { schen beiden Polen manövrieren. }\end{array}$ \\
\hline Geographie & $\begin{array}{l}\text { ie schiere Grösse der EU führt zu einem variierenden Bedrohungsempfin- } \\
\text { den. Z.B. sehen östliche Länder Russland als Bedrohung, während westliche } \\
\text { Länder stärker die russische Position als Partner hervorheben. }\end{array}$ \\
\hline
\end{tabular}

Tabelle 3: Ursachen für divergierende Rollenkonzepte (eigene Darstellung).

94 Quellen von Rollenkonzepten identifizieren u.a. Wish, Naomi: National Attributes as Sources of National Role Conceptions: A Capability-Motivation Model, in: Walker, Stephen G. (Hg.): Role Theory and Foreign Policy Analysis, Durham 1987, 94-103 und Sampson III, Martin/Walker, Stephen G.: Cultural Norms and National Roles: A Comparison of Japan and France, in: Walker, Stephen G. (Hg.): Role Theory and Foreign Policy Analysis, Durham 1987, 105-122. 
Diese und andere Faktoren beeinflussen die aussenpolitischen Rollenkonzepte der Staaten. In der Folge weisen sie divergierende Erwartungen an die Bedeutung der GSVP auf: "Nach wie vor gibt es keine gemeinsame europäische strategische Kultur. "95 Unterschiedliche Rollenkonzepte erschweren somit die Zusammenarbeit in der Sicherheits- und Verteidigungspolitik. Sie formen einen Rahmen, in dem sich die GSVP-Politiken der Mitgliedstaaten bewegen, und beeinflussen den Grad an sicherheits- und verteidigungspolitischer actorness, den ein Mitgliedstaat der EU zuschreibt. Rollenkonzepte sind grundsätzlich stabil, so dass ein Wandel und damit auch eine Annäherung der Rollen viel Zeit benötigt:

„[...] [D]ie Hoffnungen [haben sich] nicht erfüllt, dass die Zusammenarbeit in GSVP-Operationen die sicherheits- und verteidigungspolitischen Sichtweisen der EU-Mitgliedstaaten zusehends konvergieren lässt. Im Gegenteil: Eine gemeinsame europäische, strategische Kultur' ist auch zehn Jahre nach der ersten GSVP-Mission in weiter Ferne." 96

Beispielhaft werden die Konsequenzen, die aus unterschiedlichen Rollenkonzepten resultieren, anhand einer kurzen Fallstudie zur Bundesrepublik Deutschland dargestellt.

Deutschland könnte als einwohnerstärkstes, geographisch zentrales und ökonomisch starkes Land die GSVP entscheidend voranbringen. Stattdessen lassen sich mit diesem Fallbeispiel im Rollenkonzept verwurzelte Gründe für eine zurückhaltende GSVP-Politik sowie für Vertrauens- und Verständigungsprobleme aufzeigen, die aus einer unterschiedlichen Eigenwahrnehmung des ego und Fremderwartung des alter resultieren.

Deutschland war am Gipfel von Saint Malo, der die Gründung der GSVP anstiess, nicht beteiligt. Auch wenn sich das Land später in verschiedene Initiativen zur Weiterentwicklung der GSVP einbrachte, so kann doch nicht von einer Position als driver gesprochen werden; vor allem in Missionen agiert das Land zurückhaltend. Eingebettet ist die deutsche GSVP-Politik in ein aussenpolitisches Rollenkonzept, das Kirste/Maull als "Zivilmachtskonzept" bezeichnen:

"Eine Zivilmacht ist ein Staat, dessen außenpolitisches Rollenkonzept und Rollenverhalten gebunden sind an Zielsetzungen, Werte, Prinzipien sowie Formen der Einflußnahme und Instrumente der Machtausübung, die einer Zivilisierung der internationalen Beziehungen dienen."97

Zivilmächte streben unter anderem nach multilateralen Kooperationen, fördern die Bildung von Instituti-

95 Wientzek (2013), 4

96 Von Ondarza/Overhaus (2013), 2.

97 Kirste, Knut/Maull, Hanns W.: Zivilmacht und Rollentheorie, in: Zeitschriff für Internationale Beziehungen 3 (1996) 2, 300. 
onen und tragen Konflikte nur in Ausnahmen mit militärischer Gewalt aus. ${ }^{98}$ In Deutschland spiegelt sich das Zivilmachtskonzept unter anderem in einer ,Kultur der Zurückhaltung' wider. Die Bevölkerung steht Auslandseinsätzen skeptisch gegenüber. Fragen der Sicherheits- und Verteidigungspolitik erfahren zudem tendenziell wenig Aufmerksamkeit. Auch verfügt das Land über wesentlich geringere militärische Fähigkeiten als die anderen grösseren EU-Staaten Frankreich und Grossbritannien. Insgesamt präsentiert sich Deutschland sicherheits- und verteidigungspolitisch als ein ,kleiner' unter den grossen Mitgliedstaaten und somit anders als in der Wirtschaftspolitik.

Die Skepsis der deutschen Bevölkerung zu Einsätzen der Bundeswehr, die Zurückhaltung bei Auslandseinsätzen oder das Parlamentsbeteiligungsgesetz stossen auf gegenläufige Erwartungen der Partner: Diese gehen zunehmend von einer stärkeren Übernahme von Verantwortung durch die Bundesrepublik aus. Gleichzeitig können sie ,Eigenarten' wie die deutsche Zurückhaltung oder die starke Beteiligung des Bundestags auf Grund eigener abweichender Rollenkonzepte teils nicht nachvollziehen. ${ }^{99}$

Berlin laviert daher zwischen dem eigenen Selbstbild und den Erwartungen, die von aussen an Deutschland gerichtet werden. Dies begünstigt ein zauderndes und zögerliches Vorgehen der Bundesrepublik und eine teils mangelnde Kohärenz deutscher Aussenpolitik. So enthielt sich Deutschland anders als seine Partner bei der Libyen-Abstimmung im UN-Sicherheitsrat, stimmte dann einem Einsatz der NATO zu, zog anschliessend die eigenen Soldaten aus den AWACS-Flugzeugen ab und beliess gleichzeitig 103 deutsche Offiziere und Unteroffiziere in den Stäben der NATO, die unter anderem an der Auswahl militärischer Ziele beteiligt waren. Solche Inkohärenzen führen zu einem Mangel an Glaubwürdigkeit. Das Vertraven der Partner leidet.

"Die deutsche Enthaltung zu Libyen im UN-Sicherheitsrat wiegt immer noch schwer bei den Partnern in Europa. Es herrscht bestenfalls Verwirrung über die Berliner Außen- und Sicherheitspolitik, schlimmstenfalls wird Deutschland unterstellt, dass es sich aus diesem Politikfeld der EU ganz verabschiedet hat. So gilt Deutschland als schwer einschätzbar und getrieben von seinen wirtschaftlichen Interessen." 100

Grundlegende Komponenten der GSVP wie das P\&S basieren jedoch auf Verlässlichkeit. Verzichten Staaten auf militärische Fähigkeiten, müssen sie sicher sein, dass die Partner, die über diese Fähigkeiten

98 Kirste/Maull (1996), 290-293.

99 Vgl. Neukirch, Ralf/Repinski, Gordon: Schlechter Geschmack, in: Der Spiegel, 4/2013, 25; Bittner, Jochen/Geis, Matthias/ Lau, Jörg/Ulrich, Bernd/Wurmb-Seibel, Ronja: Wir tun doch nix..., in: Zeit online, 21.03.2013, URL: http://www.zeit.de/2013/13/ Deutschland-Aussenpolitik (22.03.2013).

100 Keller et al (2012), 80. Vgl. auch Zarandi (2013), 4; Winter (2013), 7; AK Internationale Sicherheit (2012), 7-8. 
verfügen, diese im Fall der Fälle zur Verfügung stellen. Hier sorgt der Parlamentsvorbehalt für Irritationen.

„Kritiker bemängeln zu Recht, dass glaubwürdige Sharing-Arrangements mit unseren europäischen Partnern nicht getroffen werden können, solange deren Einsatz - und sei es nur durch die Partner - dem Vorbehalt des Bundestags unterliegen. Erst 2011 hat die Debatte um den Einsatz von AWACS-Einheiten in Afghanistan dafür ein Beispiel geliefert. Die Verteidiger des Vorbehalts verweisen zwar darauf, dass der Bundestag noch nie ein von der Regierung gewünschtes Mandat verweigert hat. Das ändert jedoch nichts an der Wahrnehmung unserer Partner [...]." ${ }^{101}$

Obwohl das Rollenkonzept der Zivilmacht Deutschland dem der EU näher steht, als dies bei den meisten anderen Mitgliedstaaten der Fall ist, ist es für die Bundesrepublik schwierig, der EU eine ausgeprägte sicherheits- und verteidigungspolitische actorness zuzuerkennen und gleichzeitig dem eigenen Selbstbild sowie den Erwartungen von aussen gerecht zu werden. Diese Erwartungen entspringen teils den Rollenkonzepten der Mitgliedstaaten, die dem deutschen Rollenkonzept in wichtigen Punkten entgegenlaufen. So stellt Heyer im Kontext des französischen Einsatzes in Zentralafrika beispielsweise fest: "Paris und Berlin - das sind auch in der Außen- und Sicherheitspolitik zwei Pole, die sich gegenseitig abstoßen."102 Treffen die unterschiedlichen Rollenkonzepte der Mitgliedstaaten in der GSVP aufeinander, so sind die Grundlagen für auseinanderdriftende Ziele, Prioritäten und Konzepte in der GSVP ebenso gelegt wie für fehlendes Vertrauen, das den Transfer von Souveränität an die EU erschwert. Dies schwächt in der Folge die Handlungsfähigkeit der GSVP.

\subsection{Zwischenfazit}

Insgesamt lassen sich in allen vier Kategorien der actorness Probleme feststellen. Viele der Herausforderungen in den Komponenten Kohärenz, Autorität und Autonomie sind mit der Bedeutung verbunden, die die Mitgliedstaaten nationaler Souveränität beimessen. Daneben bestehen auch Probleme auf EU-Ebene (institutionelle Probleme) und im Verhältnis zur NATO. Den Kern des Problems bilden jedoch die divergierenden aussenpolitischen Rollenkonzepte der Mitgliedstaaten. Diese führen zu einem mangelnden Verständnis für andere sicherheits- und verteidigungspolitische Traditionen und Einstellungen und können - wie im Falle Deutschlands - ein Auseinanderklaffen von Eigen- und Fremderwartungen auslösen. Nationale Rollenkonzepte sind zudem Quelle für ein nicht umfassend ausgeprägtes Vertrauen in andere Mitgliedstaaten, was wiederum den Souveränitätstransfer und die Zusammenarbeit in der GSVP erschwert.

\footnotetext{
101 Keller et al (2012), 86.
}

102 Heyer et al (2013), 86. 


\section{Reaktionen}

Wie aber kann mit den oben aufgezeigten Herausforderungen umgegangen werden? Im Folgenden wird zunächst kurz auf den Dezembergipfel 2013 der Staats- und Regierungschefs eingegangen, bevor im Anschluss mögliche Reaktionen aufgezeigt werden.

\subsection{Der Dezembergipfel 2013}

Am 19. und 20. Dezember 2013 stand das Thema GSVP erstmals seit fünf Jahren wieder auf der Agenda des Europäischen Rats. Dies und die vorhergehenden Berichte der Europäischen Kommission und der Hohen Vertreterin sowie ein Treffen der Verteidigungsminister im September 2013 weckten im Voraus teils hohe Erwartungen an die Ergebnisse der Gespräche: Könnte der Gipfel zu einem Neustart für die GSVP führen? ${ }^{103}$ Die Mehrzahl der Beobachter relativierte diese Hoffnungen jedoch. Allein die Tatsache, dass die GSVP auf der Tagesordnung stehe, sei als Erfolg zu werten und könne Anstösse für eine Weiterentwicklung geben. Zu erwarten seien eher kleine Fortschritte, zum Beispiel bei den Battle Groups. ${ }^{104}$ Gedämpft wurden die Erwartungen zusätzlich dadurch, dass aktuelle Themen wie die Schaffung einer Bankenunion auf die Tagesordnung drängten. ${ }^{105}$

Diskutiert werden sollten drei Ziele: die Steigerung der Effektivität, der Sichtbarkeit und des Einflusses der GSVP, die Stärkung der Verteidigungstähigkeiten und die Stärkung der europäischen Rüstungsindustrie. 100 Hierauf aufbauend bestimmten zahlreiche Einzelvorschläge die Debatte im Vorfeld des Gipfels. Auf der Tagesordnung fanden sich schliesslich drei Themenkomplexe wieder: P\&S, die Prozesse und Institutionen im Krisenmanagement sowie die Rüstungsindustrie. Wie zu erwarten bezogen sich die Beschlüsse des Gipfels vornehmlich auf eine engere Zusammenarbeit in Fragen der Rüstungsindustrie. Beschlossen wurden zudem eine Kooperation bei vier Projekten zu gemeinsamen Drohnen, bei der Anschaffung von Flugzeugen zur Luftbetankung und der nächsten Generation Satellitentechnologie sowie ein gemeinsames Training im Bereich Cyber Defence. Die Berichterstattung der Medien bestimmte allerdings ein französischer Vorstoss im Vorfeld des Gipfels. Präsident François Hollande hatte angekündigt, dass der französische Einsatz in Zentralafrika keine Kosten für sein Land verursachen, sondern aus einem Entwick-

103 Teils wurde gar von einem "make or break" ausgegangen. Iso-Markku (2013), 3.

104 Eine solche eher zurückhaltende Position findet sich beispielsweise bei Iso-Markku (2013), 3. Vgl. auch Linnenkamp/Mölling (2013), 2

105 Vgl. Frontini, Andrea: Will the December Summit Provide a coup de théâtre in European Defence? European Policy Centre, Commentary, 17.12.2013, 1.

106 Vgl. Iso-Markku (2013), 3; Arteaga, Félix: El Consejo Europeo de diciembre de 2013: repercusiones para la industria y la defensa de España, Real Institute Elcano, ARI 46/2013, 27. November 2013, 1. 
lungsfonds der EU bezahlt werde. Zusätzlich forderte er die Einführung eines ständigen Fonds für die Finanzierung von Auslandseinsätzen, was von anderen Mitgliedstaaten scharf abgelehnt wurde. ${ }^{107}$

Zusammenfassend sind die Gipfelergebnisse kein grosser Wurf. Dieser war angesichts des Raums, den die Finanzkrise weiterhin einnimmt, und des Unwillens der ,Grossen Drei' zu einem neuen Vorstoss zur Stärkung der GSVP auch nicht zu erwarten. ${ }^{108}$ Allerdings hat der Gipfel neue Projekte angestossen und ein Signal gesetzt: "Mit dem Europäischen Ratsgipfel eröffnet sich eine Gelegenheit, die GSVP zur Chefsache zu machen und ihr eine neue Dynamik zu verleihen."109

\subsection{Reformvorschläge}

Wie können die EU und ihre Mitgliedstaaten auf die oben analysierten Herausforderungen reagieren? Im Folgenden werden abschliessend ausgewählte Reformvorschläge aus den analysierten Publikationen vorgestellt und im Spiegel der theoretischen Ergebnisse bewertet.

Häufig werden Überlegungen zu den Grundlagen der GSVP als Notwendigkeit angesehen, das heisst zu ihrer Rolle und ihrer strategischen Vision. Ziele sollen klar definiert und Handlungsbereiche festgelegt werden. ${ }^{110}$ "[...] [D]ie GSVP braucht eine grundsätzliche Neuausrichtung." ${ }^{11}$ So sieht Biscop drei wichtige Verantwortungen der GSVP: die Übernahme von Führung bei der Sicherstellung von Sicherheit und Frieden in Europas weiterer Nachbarschaft, Beiträge zur globalen maritimen Sicherheit sowie zum kollektiven Sicherheitssystem der UNO. ${ }^{12}$ Niedergeschrieben werden könnten die Grundlagen in einem

107 Vgl. Spiegel online: Frankreichs Präsident Hollande fordert EU-Fonds für Militäreinsätze, 13.12.2013, URL: http://www.spiegel. $\mathrm{de} /$ politik/ausland/frankreichs-praesident-hollande-fordert-eu-fonds-fuer-militaereinsaetze-a-938940.html (28.01.2014); Weiland, Severin/Gebauer, Matthias: EU-Gelder für Afrika-Einsatz. Hollandes Vorstoß verblüfft Brüssel und Berlin, 10.12.2013, URL: http://www.spiegel.de/politik/deutschland/berlin-reagiert-zurueckhaltend-auf-truppenplan-von-hollande-a-938282.html (28.01.2014); Rettman, Andrew: France, Germany and UK Show Discord on EU Defence, in: EUobserver, 20.12.2013, URL: http://euobserver.com/defence/122570 (28.01.2014).

108 Während Grossbritannien mit einem grundsätzlich europaskeptischen Kurs keinen Vorstoss in diese Richtung unternehmen kann, war die neue deutsche Bundesregierung erst kurze Zeit im Amt. Für das wirtschaftlich angeschlagene Frankreich demgegenüber ist die Rechtfertigung teurer Militäreinsätze problematisch geworden, was zu dem oben genannten Wunsch nach einer stärkeren gemeinsamen Finanzierung führte. Vgl. zu den Positionen Frankreichs und Grossbritanniens Dufour, Nathan/Parkes, Roderick: Will France squander its lead on European defence?, in: EUobserver, 18.12.2013, URL: http://euobserver.com/defence/122543 (28.01.2014). Auch zu anderen Mitgliedstaaten existieren Darstellungen: So analysiert Arteaga vor dem Hintergrund des Gipfels Auswirkungen der GSVP auf Spanien und schlägt Handlungsmöglichkeiten vor. Vgl. Arteaga (2013).

109 Wientzek (2013), 5.

110 Vgl. Hopia (2013), 50-53; AK Internationale Sicherheitspolitik (2012), 5-7; Wientzek (2013), 6-7.

111 Von Ondarza/Overhaus (2013), 2.

112 Vgl. Biscop, Sven: And What Will Europe Do? The European Council and Military Strategy, Royal Institute for International Relations, Security Policy Brief, 46, Mai 2013, 2-5. 
Weissbuch oder einer überarbeiteten ESS, unterstützł durch regelmässige Verteidigungsgipfel. ${ }^{113}$

Im Blickpunkt institutioneller Vorschläge steht eine Stärkung der Europäischen Verteidigungsagentur. Der EDA-Lenkungsausschuss könnte so die Stelle für Initiativen zur gemeinsamen Planung und Beschaffung von Rüstungsgütern werden. ${ }^{114}$ Gefordert wird zudem eine Ausweitung der Finanzmittel der EDA, vor allem für gemeinsame Projekte der Mitgliedstaaten, aber auch eine stärkere Rolle der Kommission im Rüstungsmarkt. ${ }^{115}$ Weitere institutionelle Forderungen beinhalten die Schaffung eigener Planungsstrukturen für die GSVP, die Schaffung einer interparlamentarischen Versammlung von Vertretern aus den nationalen Parlamenten und dem Europäischen Parlament sowie eine stärkere Vergemeinschaftung. ${ }^{116}$

Allgemein wird eine engere Zusammenarbeit von GSVP und NATO gewünscht. Sie sollten nicht als Konkurrenten betrachtet werden, vielmehr müsse ihre Arbeitsteilung diskutiert werden: „Konkret bedeutet das, die Stärkung des europäischen Pfeilers innerhalb der Allianz und die Herausbildung einer EU-Sicherheitsund Verteidigungspolitik als komplementäre Prozesse zu begreifen. "117 So könnten ähnliche Ansätze in GSVP und NATO aufeinander abgestimmt werden: „Eine Verbindung der Prozesse von Pooling und Sharing und Smart Defence muss die zentrale Achse jeglicher Verteidigungskooperation in Europa sein."118 Eine Verbesserung der Situation scheint jedoch nur bei einem Ende der türkisch-zypriotisch-griechischen Blockaden möglich. ${ }^{119}$

Darüber hinaus werden Reformvorschläge im Bereich Missionen und Operationen genannt. Angedacht wird unter anderem eine Reform des Athena-Mechanismus, durch die mehr Kosten gemeinsam getragen werden könnten. ${ }^{120}$ Insbesondere aber könnten die Wirksamkeit der Missionen stärker überprüft und die Voraussetzungen für Missionen genauer definiert werden. So schlagen von Ondarza/Overhaus vor, dass Missionen nur dann durchgeführt werden, wenn die Interessen mehrerer Mitgliedstaaten betroffen sind und die Möglichkeit besteht, dass die EU tatsächlich wirksam zur Krisenbewältigung beitragen kann. ${ }^{121}$

113 Vgl. Wientzek (2013), 6-7; von Ondarza/Overhaus (2013), 3-4; Iso-Markku (2013), 4-5; Keller et al (2012), 81 ; AK Internationale Sicherheitspolitik (2012), 5-7. Befürchtet wird, dass eine Überarbeitung die Unterschiede deutlich machen und das Dokument weniger ambitioniert und spezifisch würde als die ESS.

114 Vgl. Keller et al (2012), 86-87; Zarandi (2013), 7.

115 Vgl. Wientzek (2013), 6-7; Maulny (2013). Hinzu kommen weitere Vorschläge wie jener, dass die EDA Berichte über die, verteidigungstechnologische und -industrielle Basis Europas' für den Europäischen Rat erstellen könnte. Vgl. Linnenkamp/Mölling (2013), 3.

116 Vgl. Wientzek (2013), 6-7; AK Internationale Sicherheitspolitik.

117 Keller et al (2012), 82. Vgl. auch von Ondarza/Overhaus (2013), 3.

118 Zarandi (2013), 8.

119 Vgl. Hopia (2013), 50-53.

120 Vgl. Wientzek (2013), 6-7.

$121 \mathrm{Vgl}$. von Ondarza/Overhaus (2013), 2. 
Schliesslich werden Instrumente stark diskutiert, die zwar im Vertrag von Lissabon vorgesehen, bisher aber nahezu ungenutz† geblieben sind: die SSZ, die Battle Groups sowie die Solidaritäts- und Beistandsklauseln. Gefordert wird daher eine Evaluierung dieser Instrumente. ${ }^{122}$ "Die EU-Staaten werden mit dem Gipfel die GSVP nicht revolutionieren. Sie sollten aber die Chance ergreifen, bereits getroffene Entscheidungen zur GSVP endlich umzusetzen und das weiter auszubauen, was bereits funktioniert." 123 Im Umfeld der SSZ gelten sogenannte Insellösungen als möglicher Reformschritt. Hier könnten Staaten mit ähnlichen sicherheits- und verteidigungspolitischen Orientierungen Inseln der Kooperation bilden. ${ }^{124}$ Bereits existierende Kooperationen wie die Nordic Defence Cooperation (NORDEFCO) und die britisch-französische Zusammenarbeit könnten sich hier einfügen. ${ }^{125}$ Zarandi weist darauf hin, dass eine Zusammenarbeit mit nur einer geringen Zahl von Partnern auch das Problem der Souveränitätsabgabe verringern könnte: "Mit Inseln werden verbunden: Übersichtlichkeit, Begrenzung, Vertrauen und Zusammenhalt." ${ }^{126}$ Die EU müsste bei solchen Insellösungen sicherstellen, dass die Kooperationen bei der Verkleinerung von Fähigkeitslücken helfen. ${ }^{127}$

Bei den noch nie eingesetzten Battle Groups sind die meisten Reformvorschläge grundsätzlicher Art: Die Idee soll diskutiert und neu belebt werden. ${ }^{128}$ Als sinnvoll wird eine längerfristige und nach regionalen Gesichtspunkten gestaltete Zusammenarbeit von Staaten erachtet. ${ }^{129}$ Dadurch würden die Battle Groups mit den Insellösungen verknüpft. „Die Battlegroups bleiben ein wichtiges Instrument der europäischen Verteidigungskooperation und müssen im Rahmen der Inselkooperation weiter gestärkt werden. " ${ }^{30}$ Zudem könnten die Battle Groups nicht nur in GSVP-Missionen einsetzbar sein, sondern auch für NATO- und UNO-Missionen zur Verfügung stehen. ${ }^{131}$

Schliesslich behandeln viele Ideen die Probleme der Finanzierung der Fähigkeiten und hiermit verbunden

122 Vgl. von Ondarza/Overhaus (2013), 3-4; Iso-Markku (2013), 4-5; Hopia (2013), 50-53.

123 Linnenkamp/Mölling (2013), 2.

124 Vgl. von Ondarza/Overhaus (2013), 2; Keller et al (2012), 84; O’Donnell (2013), 3-4.

125 Zu nennen sind zudem die Kooperationen von Frankreich, Spanien, Italien und Portugal (EUROFOR-Gruppe), der Visegrád-Staaten sowie der HELBROC-Gruppe (Griechenland, Bulgarien, Rumänien, Zypern). Deutschland hat seinerseits Ende Mai 2013 Absichtserklärungen zur bilateralen Zusammenarbeit mit Polen und den Niederlanden unterzeichnet. Vgl. Zarandi (2013), 4-6. Vgl. auch Camporini/ Briano (2013), 7-9.

126 Zarandi (2013), 5.

127 Vgl. Iso-Markku (2013), 7.

128 Vgl. Wientzek (2013), 6-7; Zarandi (2013), 7.

129 Vgl. von Ondarza/Overhaus (2013), 3; Zarandi (2013), 7.

130 Zarandi (2013), 7.

131 Vgl. von Ondarza/Overhaus (2013), 3; Zarandi (2013), 7. 
das $\underline{P \& S}$ und den Rüstungsmarkt. „Bei unveränderten Streitkräftestrukturen und Beschaffungsverfahren wird der Spardruck zwangsläufig zum Abbau militärischer Fähigkeiten in Europa führen. Die europäische Handlungsfähigkeit wird damit weiter eingeschränkt." ${ }^{132}$ Die Voraussetzungen für eine bessere Kooperation könnte ein European Defence Review schaffen, der eine Bestandsaufnahme der vorhandenen Fähigkeiten ermöglicht. ${ }^{133}$ Dies könnte zu einer besseren Abstimmung der finanziellen Planungen führen. Gefordert wird zudem, dass die Staaten eine gemeinsame Richtung für die Ausgaben sowie Mechanismen zur Harmonisierung der Haushalte entwickeln, die einen effizienten Einsatz der Mittel erleichtern. ${ }^{134}$ Eine wichtige Rolle beim Abbau von Fähigkeitslücken in Zeiten knapper Kassen soll ein Ausbau des P\&S spielen, beispielsweise im strategischen Lufttransport. ${ }^{135}$ Linnenkamp/Mölling empfehlen, hier zunächs† Flaggschiffprojekte zu lancieren, die als ,Kooperationstreiber' fungieren könnten, zum Beispiel ein europäisches Luftüberwachungsgeschwader. ${ }^{136}$ Vorgeschlagen werden schliesslich auch der Abbau der Fragmentierung des Rüstungsmarktes und die Schaffung eines echten Binnenmarktes für Rüstungsgüter. ${ }^{137}$

\subsection{Zwischenfazit: Bewertung der Reformvorschläge im Spiegel der Theorie}

Die vorgestellten Reformideen bestehen einerseits aus Vorschlägen für eine grundlegende Neubestimmung der GSVP und andererseits aus Ideen zur Lösung einzelner Herausforderungen. Alle könnten durchaus positiv auf die sicherheits- und verteidigungspolitische actorness der EU und damit auf die Handlungsfähigkeit der GSVP wirken. Die obige Analyse der Souveränitäts- und Rollenkonzepte führt jedoch zu folgenden Annahmen:

- Einige Vorschläge erfordern die Abgabe von Souveränität durch die Mitgliedstaaten und sind daher schwierig einzuführen (zum Beispiel Verstärkung des P\&S).

- Einige Vorschläge könnten Widerstand auslösen, der aus den nationalen Rollenkonzepten resultiert (zum Beispiel unterschiedliche Vorstellungen zu den Zielen, Strategien, Prioritäten).

132 Keller et al (2012), 84.

133 Vgl. Linnenkamp/Mölling (2013), 2-3.

134 Vgl. Wientzek (2013), 6-7; Iso-Markku (2013), 6-7.

135 Vgl. Wientzek (2013), 6-7.

136 Vgl. Linnenkamp/Mölling (2013), 3-4. Häufig erwähnt wird auch die Zusammenarbeit bei Drohnen. So weist O'Donnell darauf hin, dass verschiedene Staaten über gemeinsame Pläne verfügen: Frankreich und Grossbritannien beispielsweise für ein bilaterales Programm. Ebenso bestehen Pläne für eine Kooperation von EADS und der italienischen Finmeccanica sowie für eine deutsch-französische Kooperation. Vgl. O'Donnell, Clara Marina: Time to Bite the Bullet on European Defence, CER-Blogg, 01.02.2013, URL: http://centreforeuropeanreform. blogspot.de/2013/02/time-to-bite-bullet-on-european-defence.html (27.01.2014).

137 Vgl. Iso-Markku (2013), 7; Wientzek (2013), 6-7. Bisher berufen sich die Regierungen meist auf eine Ausnahmeklausel im EUVertrag. Demnach sind Wettbewerbseinschränkungen möglich, wenn die nationale Sicherheit betroffen ist. 
Bereits der Beschluss solcher oder ähnlicher Reformen erscheint daher nicht unkompliziert; werden sie tatsächlich eingeführt, kann es gegebenenfalls, wie bereits aktuell bei den Battle Groups oder der Bündnisklausel, zu Problemen bei der Umsetzung kommen.

Gleichzeitig versuchen einige Vorschläge bereits, diese Schwierigkeiten einzubeziehen. So könnten sich bei Insellösungen (und damit verbunden bei reformierten Battle Groups) Staaten mit ähnlichen Rollenkonzepten zusammenfinden. Dies könnte die Abgabe von Souveränität erleichtern. Die EU müsste bei solchen Insellösungen jedoch darauf achten, dass die GSVP als Dach fungiert und Insellösungen koordiniert durchgeführt werden. Ist dies der Fall, könnte die sicherheits- und verteidigungspolitische Handlungsfähigkeit der EU gestärkt werden.

\section{Schlussfolgerungen}

Im Umfeld des Dezembergipfels 2013 des Europäischen Rats diagnostizierten Kommentatoren eine Krise der GSVP. Auch das vorliegende Paper bestätigt diesen Befund und zeigt eine grosse Zahl von Herausforderungen auf. Wie aber lässt sich diese Krise verstehen? Wo liegen ihre Wurzeln? Angenommen wurde, dass es der EU an einer ausgeprägten sicherheits- und verteidigungspolitischen actorness mangelt. Tatsächlich konnte gezeigt werden, dass in allen vier Bereichen der sicherheits- und verteidigungspolitischen actorness Probleme auftreten und hierdurch die Handlungsfähigkeit der EU begrenzt ist. Der actorness-Ansatz hat sich für die Analyse der Probleme der GSVP als zielführend erwiesen. Er kann dieses Politikfeld, das stark intergouvernemental geprägt ist, aber auch durch supranationale Institutionen beeinflusst wird, besser erfassen, als die Perspektiven einer, Sammlung von Staaten' oder einer (entstehenden) polity es erlauben.

Es fällt auf, dass viele der analysierten Herausforderungen ihre Ursache auf der nationalen Ebene finden: Die Mitgliedstaaten verfolgen in der GSVP unterschiedliche Ziele, Strategien und Prioritäten, sie stimmen ihre Verteidigungshaushalte und Anschaffungen nicht ausreichend aufeinander ab, bevorzugen unterschiedliche Missionen, versuchen ihre Rüstungsindustrie zu schützen und meiden P\&S-Initiativen. Gezeigt werden konnte, dass die Bedeutung nationaler Souveränität und die divergierenden aussenpolitischen Rollenkonzepte der Mitgliedstaaten eine bessere Zusammenarbeit behindern. „Denn das Grundproblem der GSVP kann niemand aus der Welt schaffen: Die 27 Mitgliedstaaten der EU haben sehr unterschiedliche Vorstellungen über ihre Sicherheitspolitik und ihre Souveränität in Fragen von Krieg und Frieden." 138

\footnotetext{
138 Winter $(2013), 7$.
} 
Sicherheit und Verteidigung ist weiterhin ein Feld, in dem die Mitgliedstaaten zögern, nationale Souveränität abzugeben. Zusätzlich erschwert wird dies durch unterschiedliche, teils gegenläufige Rollenkonzepte der Staaten, die nicht nur zu verschiedenen Politiken innerhalb der GSVP, sondern auch zu gegenseitigem Unverständnis und mangelndem Vertrauen führen. Die Herausforderungen, die im Umfeld des Dezembergipfels festgestellt wurden, sind also ein Symptom für tiefer liegende Probleme, die nicht auf kurze Sicht gelöst werden können.

Ist es unter diesen Bedingungen somit überhaupt möglich, die Krise zu überwinden? Auch wenn die Mitgliedstaaten unterschiedliche Rollenkonzepte aufweisen, so teilen sie doch grundlegende Interessen und Werte. ${ }^{139}$ Die europäische Integration lebt von kleinen Schritten. Stärkere Kooperation in wirtschaftlichen Fragen wie dem Rüstungsmarkt könnte daher ebenso ein Schritt zu einer Vertiefung der Zusammenarbeit sein wie die angesprochenen Insellösungen, die Rücksicht auf Souveränitätsbedenken und unterschiedliche Rollenkonzepte nehmen können.

Seit 2012 hat es mehrere Treffen von Vertretern der Mitgliedstaaten und Initiativen der Mitgliedstaaten gegeben, aus denen ebenso wie aus dem Dezembergipfel weitere Fortschritte entstehen könnten. Auch Signale aus Deutschland, das anstrebt, mehr Verantwortung in der Aussenpolitik zu übernehmen, könnten sich positiv auswirken, ebenso wie externer Druck, unter anderem durch neue Sicherheitsbedrohungen, die Finanzkrise und den Rückzug der USA aus Europa. Da Rollenkonzepte relativ stabil und Sorgen über den Verlust nationaler Souveränität tief verankert sind, benötigen solche Prozesse jedoch Zeit. Ein schneller Neustart, der die GSVP aus ihrer Rolle als reines "Zusatzvergnügen" 140 zu nationaler Sicherheits- und Verteidigungspolitik und zur NATO erweckt, ist somit vorerst ebenso wenig zu erwarten wie ein grundsätzliches Scheitern der europäischen Zusammenarbeit in Sicherheits- und Verteidigungsfragen.

139 Dies wird auch in den Papers relativ selten erwähnt. Eine Ausnahme ist Biscop (2013), 2.

140 AK Internationale Sicherheitspolitik (2012), 5. 


\section{Literatur}

Algieri, Franco: Die Gemeinsame Außen- und Sicherheitspolitik der EU, Wien 2010.

Arbeitskreis Internationale Sicherheitspolitik: Ein Kompass für die GSVP, Internationale Politikanalyse, Friedrich-Ebert-Stiftung, April 2012.

Arteaga, Félix: El Consejo Europeo de diciembre de 2013: repercusiones para la industria y la defensa de España, Real Institute Elcano, ARI 46/2013, 27. November 2013.

Ashton, Catherine: Preparing the December 2013 European Council on Security and Defence, Final Report by the High Representative/Head of the EDA on the Common Security and Defence Policy, Brüssel, 15.10.2013.

Asseburg, Muriel/ Kempin, Ronja (Hg.): Die EU als strategischer Akteur in der Sicherheits- und Verteidigungspolitik? Eine systematische Bestandsaufnahme von ESVP-Missionen und -Operationen, Stiffung Wissenschaft und Politik, S32, Dezember 2009

Bickerton, Chris J./Irondelle, Bastien/ Menon, Anand: Security Co-operation beyond the Nation-State: The EU's Common Security and Defence Policy, in: Journal of Common Market Studies, 49 (2011) $1,1-21$.

Biddle, Peter: Recent Developments in Role Theory, in: Annual Review of Sociology, 1986, 67-92.

Biscop, Sven: And What Will Europe Do? The European Council and Military Strategy, Royal Institute for International Relations, Security Policy Brief, 46, Mai 2013.

Bittner, Jochen/Geis, Matthias/Lau, Jörg/ Ulrich, Bernd/ Wurmb-Seibel, Ronja: Wir tun doch nix..., in: Zeit online, 21.03.2013, URL: http://www.zeit.de/2013/13/Deutschland-Aussenpolitik (22.03.2013).

Bonvicini, Gianni/Faleg, Giovanni: L'elemento istituzionale, in: Marrone, Alessandro/ Nones, Michele (Hg.): More Europe on Defence or No Europe, Istituto Affari Internazionali, Documenti IAI 13/03, Juni 2013, 11-17.

Camporini, Vincenzo/ Briani, Valerio: L'elemento militare, in: Marrone, Alessandro/ Nones, Michele $(H g$.$) : More Europe on Defence or No Europe, Istituto Affari Internazionali, Documenti IAl 13/03,$ Juni 2013, 6-1 1 .

Chappell, Laura: Poland in Transition: Implications for a European Security and Defence Policy, in: Contemporary Security Policy 31 (2010) 2, 225-248.

Coffey, Luke: EU Defense Integration: Undermining NATO, Transatlantic Relations, and Europe's Security, The Heritage Foundation, Backgrounder, No. 2806, 6.6.2013.

Deckstein, Dinah/ Traufetter, Gerald: Feld voller Feinde, in: Der Spiegel, 41 /2012, 82-83.

Diedrichs, Udo: Die Gemeinsame Sicherheits- und Verteidigungspolitik der EU, Wien 2012.

Dufour, Nathan/ Parkes, Roderick: Will France squander its lead on European defence?, in: EUobserver, 18.12.2013, URL: http://evobserver.com/defence/122543 (28.01.2014).

Ehrhart, Hans-Georg (Hg.): Die Europäische Sicherheits- und Verteidigungspolitik. Positionen, Perzeptionen, Probleme, Perspektiven, Baden-Baden 2012.

European Commission: A New Deal for European Defence. Towards a More Competitive and Efficient Defence and Security Sector. Communication from the Commission to the European Parliament, the Council, the European Economic and Social Committee and the Committee of the Regions, COM (2013) 542.

Fiott, Daniel: More Competitive, More Efficient? The 2013 European Commission Defence Communication, EGMONT Royal Institute for International Relations, Security Policy Brief, No. 49, September 2013. 
Flockhart, Trine: 'Me Tarzan - You Jane': The EU and NATO and the Reversal of Roles, in: Perspectives on European Politics and Society 12 (201 1) 3, 263-282.

Franco-British Summit: Joint Declaration on European Defense, Erklärung des britischen Premierministers und des französischen Präsidenten in Saint-Malo, 04.12.1998, URL: http://www.atlanticcommunity.org/Saint-Malo\%20Declaration\%20Text.html (27.01.2014).

Frank, Cornelia: Zivilmacht trifft "instinktiven" Atlantiker: Deutschlands und Polens Interessen in der ESVP, in: Jäger, Thomas/ Dylla, Daria W. (Hg.): Deutschland und Polen. Die europäische und internationale Politik, Wiesbaden 2008, 101-122.

Frontini, Andrea: Will the December Summit Provide a coup de théâtre in European Defence? European Policy Centre, Commentary, 17.12.2013.

Gaupp, Peter: Staaten als Rollenträger. Die Rollentheorie als Analyse-Instrument von Außenpolitik und internationalen Beziehungen, Liebefeld/Bern 1983.

Giegerich, Bastian: European Security and Strategic Culture. National Responses to the EU's Security and Defence Policy, Baden-Baden 2006.

Giegerich, Bastian/ Wallace, William: Not Such a Soft Power: The External Deployment of European Forces, in: Survival 46 (2004) 2, 163-182.

Gomes, Ana: Was Eufor Libya an April fool's joke?, in: EUobserver, 13.07.20 1 1, URL: http://euobserver.com/opinion/32624 (28.01.2014).

Grevi, Giovanni/Helly, Damien/Keohane, Daniel (Hg.): European Security and Defence Policy. The First Ten Years (1 999-2000), The European Union Institute for Security Studies, Paris 2009.

Gross, Eva/ Menon, Anand: European Defence: An Inventory for the December Summit, in: dies. (Hg.): CSDP Between Internal Constraints and External Challenges, EU Institute for Security Studies, Report No. 17, Oktober 2013, 5-10.

Harnisch, Sebastian/ Stahl, Bernhard: Einleitung: EU-Außenpolitik und Actorness, in: Stahl, Bernhard/ Harnisch, Sebastian (Hg.): Vergleichende Außenpolitikforschung und nationale Identitäten. Die Europäische Union im Kosovo-Konflikt 1996-2008, Baden-Baden 2009, 15-29.

Harnisch, Sebastian/ Stahl, Bernhard: Fazit: Die identitären Möglichkeitsräume der EU-Außenpolitik, in: Stahl, Bernhard/ Harnisch, Sebastian (Hg.): Vergleichende Außenpolitikforschung und nationale Identitäten. Die Europäische Union im Kosovo-Konflikt 1996-2008, Baden-Baden 2009, 275-292.

Hatzigeorgopoulos, Myrto/ Fara-Andrianarijaona, Lorène: EUBAM Libya: Story of a Long-awaited CSDP Mission, isis Europe, European Security Review 66, Mai 2013.

Heyer, Julia Amalia/ Puhl, Jan/ Repinski, Gordon/ Schult, Christoph: Rechnung fürs Nichtstun, in: Der Spiegel $51 / 2013,86-87$.

Hill, Christopher: The Actors in Europe's Foreign Policy, London 1996.

Holsti, Kal J.: National Role Conceptions in the Study of Foreign Policy, in: International Studies Quarterly, 14 (1970) 3, 233-309.

Hopia, Henna: Breaking Down the Walls: Improving EU-NATO Relations, Centre for European Studies 2013.

ISIS Europe: CSDP-Map, URL: http:/ /www.csdpmap.eu/mission-chart (27.01.2014), Brüssel.

Iso-Markku, Tuomas: European Defence under Scrutiny, The Finnish Institute of International Affairs, FIIS Briefing Paper 145, November 2013.

Jönsson, Christer/ Westerlund, Ulf: Role Theory in Foreign Policy Analysis, in: Jönsson, Christer (Hg.): Cognitive Dynamics and International Politics, London 1982, 122-157.

Jupille, Joseph/ Caporaso, James A.: States, Agency, and Rules: The European Union in Global Environ- 
mental Politics, in: Rhodes, Carolyn (Hg.): The European Union in the World Community, Boulder/ London 1998, $213-230$.

Keller, Patrick/Möller, Almut/Sinjen, Svenja/ Varwick, Johannes: Zivilmacht mit Zähnen. Deutsche Vorschläge für eine Neubelebung der GSVP, in: Internationale Politik, März/April 2012.

Kirste, Knut/ Maull, Hanns W.: Zivilmacht und Rollentheorie, in: Zeitschrift für Internationale Beziehungen 3 (1996) 2, 283-312.

Larsen, Henrik: Danish CFSP Policy in the Post-Cold War Period: Continuity or Change?, in: Cooperation and Conflict, 35 (2000) 1, 37-63.

Linnenkamp, Hilmar/ Mölling, Christian: Eine Agenda für den Europäischen Verteidigungsrat 2013, Stiftung Wissenschaft und Politik, SWP-Aktuell 57, Oktober 2013.

Maulny, Jean-Pierre: European Commission Communication on the Defence and Security Sector: Towards a Definition of a Strategic EDTIB?, Institut de Relations Internationales et Stratégiques, Les Notes de L'Iris, September 2013.

Missiroli, Antonio/ Gilli, Andrea/ Rogers, James: Enabling the Future. European Military Capabilities 201 3-2025: Challenges and Avenues, European Union Institute for Security Studies, Report No. 16, May 2013.

Morgenthau, Hans: Politics among Nations. The Struggle for Power and Peace, New York 1967.

Müller-Brandeck-Bocquet, Gisela (Hg.): Europäische Außenpolitik. GASP- und ESVP-Konzeptionen ausgewählter EU-Mitgliedstaaten, Baden-Baden 2002.

Neukirch, Ralf/ Repinski, Gordon: Schlechter Geschmack, in: Der Spiegel, 4/2013, 25.

O'Donnell, Clara Marina: Time to Bite the Bullet on European Defence, CER-Blogg, 01.02 .2013 , URL: http:/ / centreforeuropeanreform. blogspot.de/2013/02/time-to-bite-bullet-on-european-defence. html (27.01.2014).

O'Donnell, Clara Marina: The Trials and Tribulations of European Defence Co-operation, Centre for European Reform, July 2013.

Repinski, Gordon/ Schult, Christoph/ Traufetter, Gerald: Brennende Unterhosen, in: Der Spiegel, 50/2013, 30-34.

Rettman, Andrew: France, Germany and UK Show Discord on EU Defence, in: EUobserver, 20. 12.2013, URL: http://euobserver.com/defence/122570 (28.01.2014).

Rynning, Sten: Realism and the Common Security and Defence Policy, in: Journal of Common Market Studies 49 (201 1) 1, 23-42.

Sampson III, Martin/ Walker, Stephen G.: Cultural Norms and National Roles: A Comparison of Japan and France, in: Walker, Stephen G. (Hg.): Role Theory and Foreign Policy Analysis, Durham 1987, 105-122.

Schmalz, Uwe: Deutschlands europäisierte Außenpolitik. Kontinuität und Wandel deutscher Konzepte zur EPZ und GASP, Wiesbaden 2003.

Spiegel online: Frankreichs Präsident Hollande fordert EU-Fonds für Militäreinsätze, 13.12.2013, URL: http://www.spiegel.de/politik/ausland/frankreichs-praesident-hollande-fordert-eu-fonds-fuer-militaereinsaetze-a-938940.html (28.01.2014).

Tonra, Ben: The Europeanisation of National Foreign Policy. Dutch, Danish and Irish Foreign Policy in the European Union, Aldershot 2001.

Von Ondarza, Nicolai/ Overhaus, Marco: Die GSVP nach dem Dezember-Gipfel, Stiffung Wissenschaft und Politik, SWP-Aktuell 58, Oktober 2013.

Wallace, William: Foreign and Security Policy. The Painful Path from Shadow to Substance, in: Wallace, 
Helen/ Wallace, William/ Pollack, Mark A. (Hg.): Policy-Making in the European Union, Oxford 2005, 429-456.

Weiland, Severin/ Gebaver, Matthias: EU-Gelder für Afrika-Einsatz. Hollandes Vorstoß verblüfft Brüssel und Berlin, 10.12.2013, URL: http://www.spiegel.de/politik/deutschland/berlin-reagiert-zurueckhaltend-auf-truppenplan-von-hollande-a-938282.html (28.01.2014).

Wientzek, Olaf: Letzte Hoffnung Dezembergipfel? Impulse für die Wiederbelebung der Gemeinsamen Sicherheits- und Verteidigungspolitik, Analysen \& Argumente, 127, Konrad-Adenaver-Stiftung, Berlin, September 2013

Winter, Martin: Mit Sicherheit nichts zu machen. Im Schatten der Währungskrise steuert die EU auf den Kollaps ihrer gemeinsamen Verteidigungspolitik zu, in: Süddeutsche Zeitung, 7.5.201 3, 7.

Wish, Naomi: National Attributes as Sources of National Role Conceptions: A Capability-Motivation Model, in: Walker, Stephen G. (Hg.): Role Theory and Foreign Policy Analysis, Durham 1987, 94103.

Zarandi, Maik: Europäische Insellösungen als Fundament einer Stärkung der europäischen Verteidigungsfähigkeiten?, Analysen \& Argumente, 130, Konrad-Adenauer-Stiftung, Berlin, Oktober 2013. 
www.europa.unibas.ch 